\title{
RDUS
}

Revue de DROIT

UNIVERSITÉ DE SHERBROOKE

Titre : $\quad$ LES RÈGLEMENTS À CARACTÈRE DISCRÉTIONNAIRE EN VERTU DE LA LOI SUR L'AMÉNAGEMENT ET L'URBANISME

Auteur(s): $\quad$ Marc-André LECHASSEUR

Revue: $\quad$ RDUS, 2000-2001, volume 31, numéro 1-2

Pages: $\quad$ 199-266

ISSN : $\quad 0317-9656$

Éditeur : $\quad$ Université de Sherbrooke. Faculté de droit.

URI : $\quad$ http://hdl.handle.net/11143/12337

DOI : https://doi.org/10.17118/11143/12337 
Page vide laissée intentionnellement. 


\section{LES RÈGLEMENTS À CARACTĖRE DISCRÉTIONNAIRE EN VERTU DE LA LOI SUR L'AMÉNAGEMENT ET L'URBANISME}

par Marc-André LECHASSEUR ${ }^{* *}$

\section{SOMMAIRE}

INTRODUCTION

SECTION I - LE COMITÉ CONSULTATIF D'URBANISME

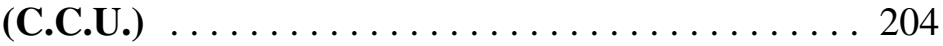

A) SON ORIGINE . . . . . . . . . . . . . . . 204

B) LA CONSTITUTiON DU COMité CONSULTATIF D'URBANISME ... . . . . . . . 205

C) CERTAINS MOTIFS D'INHABILITÉ . . . . . 207

1. Abus de confiance par un fonctionnaire public (art. 118 et 122 du Code criminel) . .......... 208

2. Actes de corruption dans les affaires municipales (art. 123 du Code criminel) . . . . . 209

3. Refus de remettre des biens (art. 337 du Code

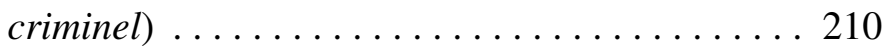

4. Faux relevé de deniers publics par un fonctionnaire public (art. 399 du Code

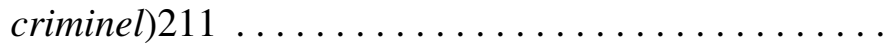

D) LES POUVOIRS DU COMITÉ CONSULTATIF

D'URBANISME ................... 212

1. Le pouvoir d'étude et de recommandation ..... 212

* Texte rédigé dans le cadre du colloque «La Loi sur l'aménagement et l'urbanisme» de la Faculté de droit de l'Université de Sherbrooke tenu le 24 février 2000.

**. L'auteur est avocat au sein du groupe de droit municipal du cabinet McCarthy Tétrault de Montréal. Il tient à remercier $\mathrm{M}^{\mathrm{e}}$ Louis Terriault, associé responsable du groupe de droit municipal, pour ses commentaires ainsi que Madame Andrée-Claude Bérubé, étudiante, pour son aide dans la préparation du présent document. 
2. Le rôle du C.C.U. dans l'application des règlements à caractère discrétionnaire . . . . . 215

E) LES RÈGLES DE RÉGIE INTERNE . . . . . . 217

SECTION II- LE RÈGLEMENT SUR LES DÉROGATIONS MINEURES . . . . . . . . . . . . . . . . . . . . . . . 219

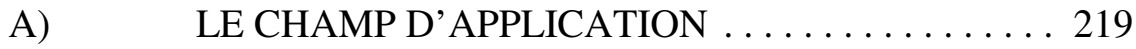

B) L'ADOPTION ET LE CONTENU DU RÈGLEMENT SUR LES DÉROGATIONS MINEURES ................... 226

C) L'ÉPOQUE DE LA PRÉSENTATION DE LA DEMANDE .................... 228

D) LA PORTÉE DU POUVOIR DISCRÉTIONNAIRE EN MATIÈRE DE DÉROGATION MINEURE . . . 229

E) LA QUANTIFICATION DE LA DÉROGATION MINEURE .................... 231

F) LE CONTRÔLE JUDICIAIRE DES DÉCISIONS DU CONSEIL MUNICIPAL . . . . . . . . . . 234

SECTION III - L ES PLA NS D’ A M É N G EM EN T

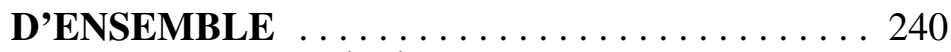

A) LE ZONAGE DIFFÉRÉ («HOLDING BY-LAW» OU «SITE PLAN BY-LAW») . . . . . . . . . . . 240

B) L'A V È NEMEN T DES PLANS D'AMÉNAGEMENT D'ENSEMBLE (P.A.E.) . . . 245

C) LES CONDITIONS D'APPROBATION D'UN PLAN D'AMÉNAGEMENT D'ENSEMBLE . . . . . 249

SECTION IV - LES PLANS D'IMPLANTATION ET D'INTÉGRATION ARCHITECTURALE

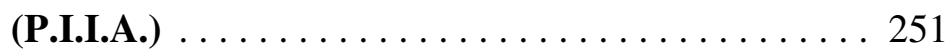

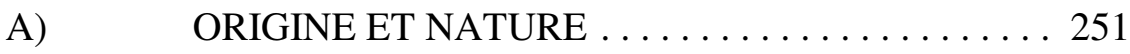

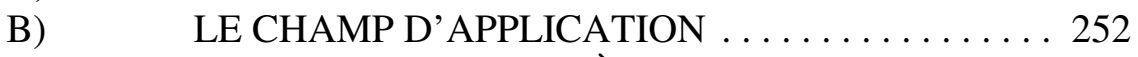

C) LE CONTENU DU RËGLEMENT SUR LES PLANS D'IMPLANTATION E T D'INTÉGRATION ARCHITECTURALE . . . . . . 254 
(2000-01) 31 R.D.U.S.

Les règlements à caractère discrétionnaire en vertu de la

Loi sur l'aménagement et l'urbanisme

SECTION V - LES ENTENTES RELATIVES À DES

TRAVAUX MUNICIPAUX $\ldots \ldots \ldots \ldots \ldots \ldots 258$

A) ORIGINE $\ldots \ldots \ldots \ldots \ldots \ldots \ldots \ldots \ldots \ldots \ldots 258$

B) LE CHAMP D'APPLICATION . ............ 259

C) LE CONTENU DU RÈGLEMENT $\ldots \ldots \ldots \ldots 260$

D) LE CONTENU DE L'ENTENTE $\ldots \ldots \ldots \ldots . . \ldots 263$

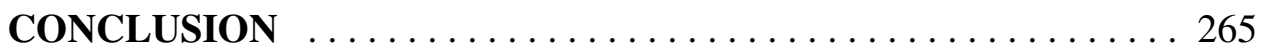





\section{INTRODUCTION}

Depuis ses débuts en 1979, la Loi sur l'aménagement et l'urbanisme ${ }^{1}$ a connu de nombreuses réformes dont certaines d'une importance non négligeable. C'est ainsi que sont successivement venues se greffer à la Loi des dispositions législatives à caractère discrétionnaire visant à amoindrir la rigidité de la réglementation d'urbanisme des municipalités pour l'adapter aux caractéristiques propres de certains sites ou aux orientations d'aménagement préconisées par ces dernières.

Par ce texte, nous avons tenté de tracer une esquisse du contenu des différents règlements à caractère discrétionnaire pouvant être adoptés par le conseil municipal dans le cadre de la gestion de l'aménagement de son territoire. Bien que peu nombreuse, la jurisprudence québécoise apporte néanmoins un certain éclairage sur la portée de cette réglementation aux contours parfois obscurs. Pour mieux la comprendre, il s'est avéré parfois nécessaire de faire l'historique des concepts ayant mené à l'adoption de ces dispositions à caractère discrétionnaire.

Il aurait été vain d'étudier les différentes facettes des dispositions législatives relatives aux dérogations mineures ${ }^{2}$, aux plans d'aménagement d'ensemble ${ }^{3}$ ainsi qu'aux plans d'implantation et d'intégration architecturale ${ }^{4}$ sans d'abord revoir les règles applicables à leur complément essentiel, le comité consultatif d'urbanisme. De la même manière, un tel survol n'aurait pas été complet sans l'analyse des articles traitant des ententes entre les promoteurs et les municipalités ${ }^{5}$.

Comme nous le verrons, la discrétion dont bénéficie le conseil municipal eu égard aux sections VI à IX du chapitre IV de la L.A.U. n'est pas absolue. À ce jour toutefois, les tribunaux se sont montrés réticents à réviser l'exercice de ce pouvoir discrétionnaire ce qui, à notre avis, ne signifie pas pour autant que

1. $\quad$ L.R.Q. c. A-19.1 [ci-après L.A.U.].

2. Ibid., art. 145.1 et s.

3. Ibid., art. 145.9 et s.

4. Ibid., art. 145.15 et s.

5. Ibid., art. 145.21 et s. 
ce dernier se trouve exempt des règles de droit administratif applicables en cette matière.

\section{SECTION I - LE COMITÉ CONSULTATIF D'URBANISME (C.C.U.)}

\section{A) SON ORIGINE}

Présent dans la L.A.U. depuis son origine, le comité consultatif d'urbanisme ${ }^{6}$ n'est pas à proprement parler une création émanant de cette loi. Bien qu'adapté aux différentes facettes de la Loi, son existence s'inspire néanmoins grandement de l'ancien régime applicable à la «commission d'urbanisme» de la Loi sur les cités et villes ${ }^{7}$ et du Code municipal du Québec ${ }^{8}$. À l'époque donc, cette commission d'urbanisme possédait des pouvoirs étendus d'étude et de recommandation en matière de construction, de zonage, d'esthétique et à l'égard du plan directeur de la municipalité, ce qui revient à dire qu'elle possédait un pouvoir général en matière d'urbanisme. En outre de ces pouvoirs, le conseil municipal pouvait lui déléguer la totalité ou certains de ses pouvoirs en ce qui a trait à l'application de la réglementation relative aux matières précitées. La jurisprudence avait d'ailleurs reconnu la possibilité que soit valablement délivré un permis de construction par la commission d'urbanisme sans que cela constitue une sous-délégation illégale de pouvoirs 9 .

Avec l'avènement de la L.A.U., les pouvoirs autrefois conférés à la commission d'urbanisme et maintenant dévolus au C.C.U. se sont vus considérablement réduits. Une délégation de pouvoirs telle que celle portant sur l'application de la réglementation d'urbanisme n'est dorénavant plus permise. Seuls les pouvoirs d'étude et de recommandation ont été maintenus.

6. [Ci-après C.C.U.]. Voir ibid., art. 146 et s.

7. L.R.Q. c. C-19 [ci-après L.C.V.].

8. $\quad$ L.R.Q. c. C-27.1 [ci-après C.M.Q.].

9. Prime c. Saint-Lambert (Town of), [1974] C.S. 536; voir aussi : Placements Lavignon Inc. c. Québec (Ville de), J.E. 79-286 (C.S.); Tremblay c. Chicoutimi (Ville de), J.E. 84-894 (C.S.); Bilodeau c. Sainte-Foy (Ville de), J.E. 85-852 (C.S.). 
Ainsi, les membres du C.C.U. ne peuvent-ils être l'objet d'un recours en mandamus puisqu'ils n'ont aucun pouvoir de délivrance de permis ${ }^{10}$.

\section{B) LA CONSTITUTION DU COMITÉ CONSULTATIF D'URBANISME}

Le conseil municipal possède le pouvoir discrétionnaire d'adopter un règlement visant à constituer un C.C.U. Ce pouvoir est prévu à l'article 146 L.A.U., qui se lit comme suit :

Article 146. Le conseil d'une municipalité peut, par règlement :

$1^{\circ}$ constituer un comité consultatif d'urbanisme composé d'au moins un membre du conseil et du nombre de membres qu'il détermine et qui sont choisis parmi les résidents du territoire;

$2^{\circ}$ attribuer à ce comité des pouvoirs d'étude et de recommandation en matière d'urbanisme, de zonage, de lotissement et de construction;

$3^{\circ}$ permettre au comité d'établir ses règles de régie interne;

$4^{\circ}$ prévoir que la durée du mandat des membres est d'au plus deux ans et qu'il est renouvelable.

Contrairement aux autres commissions et comités que peut créer le conseil municipal en vertu des pouvoirs qui lui sont dévolus, le C.C.U. nécessite la participation effective des citoyens non élus résidant sur le territoire de la municipalité. Bien que le paragraphe 1 de l'article 146 L.A.U. ne s'exprime pas clairement en ce sens, la distinction que le législateur fait entre «les membres choisis à même le conseil» et les «membres choisis parmi les résidents du territoire» nous porte à croire qu'une combinaison des deux est plus à même de refléter sa volonté.

10. Entreprises J.L. Bigras Ltée c. Sainte-Marthe-sur-le-Lac (Ville de) (23 décembre 1985), Terrebonne 700-05-000561-849 (C.S.). 
Quant au critère de résidence, il doit être interprété largement en conservant à l'esprit qu'il existe une distinction importante entre le domicile et la résidence et que la seconde se veut beaucoup moins restrictive que le premier. En effet, la notion de domicile comporte un plus grand caractère de permanence que celle de résidence. Le domicile relève de l'intention plutôt que de l'habitation réelle. Il constitue une relation juridique entre une personne et un lieu. Alors que la résidence est une question de faits, celle du domicile est une question de droit. Le domicile est donc le principal établissement d'une personne, l'endroit où elle conserve la majorité de ses intérêts financiers, économiques ou sociaux par exemple ${ }^{11}$.

Bien que le conseil municipal puisse choisir d'y être représenté par plus d'un membre, il n'existe pas de dispositions déterminant le nombre minimum de membres du C.C.U. Toutefois, vu la conjonction exercée par le «et» entre «d'au moins un membre du conseil» et «du nombre de membres qu'il détermine et qui sont choisis parmi les résidents du territoire», le conseil municipal pourrait, théoriquement, décréter par règlement que le C.C.U. est formé de seulement deux membres, ce qui, dans les faits, s'avérerait contraire à toute logique guidant la tenue des séances ou assemblées constituantes. En effet, un tel choix serait de nature à empêcher toute possibilité de formuler des recommandations claires en cas de divergence d'opinion entre les membres du C.C.U. Aussi, un minimum de trois membres apparaît requis en toutes circonstances. Ces membres sont nommés par résolution du conseil municipal suivant l'article 147 L.A.U., ce qui est également le cas des officiers du C.C.U. (président, vice-président et secrétaire).

Cet article prévoit de manière plus spécifique que «[...] le conseil peut également adjoindre au Comité des personnes dont les services peuvent lui être nécessaires pour s'acquitter de ses fonctions». Il va sans dire qu'il existe d'importantes distinctions entre les membres et les personnes ressources invitées au C.C.U. Alors que les membres ont droit de vote, les personnes ressources ne l'ont pas et sont généralement des employés au service de la municipalité ou des consultants externes spécialisés en urbanisme ou, par exemple, en architecture.

11. Wadsworth c. McCord (1885-86), 12 R.C.S. 466; Bonilla c. Lefebvre, [1964] B.R. 102. 
Quant à la durée du mandat des membres du C.C.U., elle peut être fixée à au plus deux ans conformément au quatrième paragraphe de l'article 146 L.A.U. Pour sa part, la pratique recommande des nominations rotatives de manière à ce que le C.C.U. se renouvelle pour moitié à chaque année, ce qui évite une brisure trop importante dans le traitement des dossiers en cours et assure une saine gestion des orientations générales en matière d'aménagement.

Quant à la rémunération des membres du C.C.U., elle apparaît facultative puisque non spécifiquement prévue à l'article 148 L.A.U. Toutefois, il ne nous semble pas contraire à cet article d'en prévoir une lors de l'adoption du budget annuel, du moment que sont respectées les règles relatives à la rémunération des élus prévues à la Loi sur le traitement des élus municipaux ${ }^{12}$ et celles concernant les fonctionnaires qui font l'objet des règles particulières liées à leur contrat d'emploi ${ }^{13}$.

\section{C) CERTAINS MOTIFS D'INHABILITÉ}

Les membres du C.C.U. ne sont pas considérés comme des fonctionnaires de la municipalité au sens de l'article 63 L.C.V., ce qui exclut d'emblée la possibilité d'invoquer un tel motif d'inhabilité à leur égard ${ }^{14}$. Pour le reste, le C.C.U. étant un comité de la municipalité, les motifs d'inhabilité prévus à la Loi sur les élections et les référendums dans les municipalités ${ }^{15}$ s'appliquent aux membres élus du C.C.U. ${ }^{16}$

Enfin, il faut prendre garde au mot «fonctionnaire» qui ne reçoit pas, en vertu du Code criminel, la même interprétation qu'en vertu des lois civiles et de l'article 63 précité. Suivant le Code criminel $^{17}$, l'expression «fonctionnaire municipal» désigne un membre d'un conseil municipal ou une personne qui détient une charge relevant d'un gouvernement municipal. Toujours selon le

\footnotetext{
12. L.R.Q. c. T-11.001.

13. L.C.V., supra note 7, art. 116 (4) et C.M.Q., supra note 1, art. 269 (4).

14. Plante c. Bois-Tremblay, J.E. 96-1441 (C.S.); voir aussi : Brosseau c. Trudeau, [1978] C.S. 764 conf. par (19 août 1980), Montréal 500-09-001061-787 (C.A.).

$15 . \quad$ L.R.Q. c. E-2.2.

16. Gale c. Chicoutimi (Ville de), J.E. 94-769 (C.A.) [ci-après Gale].

17. L.R.C. 1985 , c. C-46, art. 123(3).
} 
Code criminel, un membre du C.C.U. pourrait donc être déclaré inhabile par application, notamment, des articles suivants dudit code. D'ailleurs, certaines infractions au Code criminel entraînent l'inhabilité, dont les suivantes :

\section{Abus de confiance par un fonctionnaire public (art. 118 et 122 du Code criminel)}

L'abus de confiance énoncé à l'article 122 du Code criminel, en plus d'être une infraction punissable d'un maximum de cinq ans d'emprisonnement, génère une inhabilité s'étendant sur une période de cinq ans.

Pour que le fonctionnaire ou l'employé qui occupe son poste devienne inhabile, il doit pour cela être condamné pour abus de confiance commis dans le cadre général de l'exécution de ses fonctions. Il n'est pas nécessaire que l'acte commis par le fonctionnaire ou employé le soit dans le cadre spécifique de ses fonctions. Il peut être simplement posé dans l'exécution de tâches liées à sa situation au sein de la municipalité. Par exemple, un fonctionnaire qui reçoit une somme d'argent pour sceller la bonne collaboration entre les parties dans le déroulement d'un dossier ${ }^{18}$ peut être condamné pour abus de confiance. Il en va de même dans les cas de transmission d'informations confidentielles par le fonctionnaire, pour le bénéfice de tiers intéressés.

Contrairement à la notion de fraude ${ }^{19}$ qui peut aussi entraîner une condamnation suivant l'article 122 du Code criminel, il n'est pas nécessaire qu'il résulte un quelconque préjudice ou un caractère de malhonnêteté, de corruption ou d'illégalité de l'acte faisant l'objet de la condamnation pour abus de confiance. Alors que la fraude comporte un élément de vénalité préjudiciable à un tiers, l'abus de confiance n'a pas nécessairement ce caractère.

En effet, un abus de confiance constitue le fait, par un fonctionnaire ou employé, de poser un acte ou une omission contraire aux obligations et devoirs de sa tâche, tel acte ou omission lui rapportant un bénéfice ou avantage, direct

18. $\quad$ Carré c. R., J.E. 89-1515 (C.A.).

19. À cet effet, voir l'arrêt $R$. c. Hébert, [1986] R.J.Q. 236 (C.A.), qui fait la distinction entre l'abus de confiance et la fraude. 
ou indirect. Par exemple, le fait qu'un maire favorise l'embauche par la municipalité de son fils et de sa nièce peut constituer un manque d'impartialité susceptible de conduire à une condamnation pour abus de confiance. À cet effet, il n'est pas dit que le maire, en posant de tels gestes, n'avait pas un intérêt indirect dans l'embauche compte tenu du lien de parenté existant entre lui, son fils et sa nièce ${ }^{20}$.

Il n'est pas nécessaire que le bénéfice ou l'avantage souhaité se concrétise pour que l'acte ou l'omission engendre une condamnation et l'inhabilité par le fait même. Enfin, le bénéfice envisagé n'a pas à être personnel au fonctionnaire ou employé. L'acte commis peut être à l'avantage d'un membre de sa famille, pour un ami, dans l'objectif de recevoir des services de la part d'une autre personne ou des sommes d'argent.

\section{Actes de corruption dans les affaires municipales (art. 123 du Code criminel)}

La corruption dans les affaires municipales énoncée à l'article $123 \mathrm{du}$ Code criminel, en plus d'être une infraction punissable d'un maximum de cinq ans d'emprisonnement, génère elle aussi une inhabilité s'étendant sur une période de cinq ans. Pour que le fonctionnaire ou l'employé qui occupe son poste devienne inhabile, il doit pour cela être condamné pour corruption dans le cadre général de l'exécution de ses fonctions.

Il y a corruption au sens de l'article 123 du Code criminel lorsqu'une personne, «le corrupteur», donne, offre ou convient de donner à un fonctionnaire municipal, soit un prêt, une récompense, un avantage ou un bénéfice de quelque nature en considération du fait par le fonctionnaire, soit de s'abstenir de voter à une réunion du conseil municipal ou d'un de ses comités, soit de voter pour ou contre une mesure, une motion ou une résolution, soit d'aider à obtenir ou à empêcher l'adoption d'une mesure, motion ou résolution, soit d'accomplir ou d'omettre d'accomplir un acte officiel. 
Il y a évidemment corruption lorsque le fonctionnaire municipal exige, accepte, offre ou convient d'accepter soit un prêt, une récompense, un avantage ou un bénéfice de quelque nature en considération de son influence dans la prise d'une décision.

La difficulté liée à cet article réside dans l'interprétation à donner au mot «fonctionnaire». Car l'article énonce sa propre définition, qui est imprécise et qui reste encore à définir.

Le paragraphe 3 de l'article 123 du Code criminel énonce que les mots «fonctionnaire municipal» désignent un membre d'un conseil municipal ou une personne qui détient une charge relevant d'un gouvernement municipal. Si nous savons tous qui sont les membres du conseil municipal, il est moins facile par contre d'identifier ceux à l'intérieur de l'organisation municipale qui détiennent une «charge relevant d'un gouvernement municipal».

En fait, il nous est impossible de donner une définition ou d'expliquer clairement cette expression. Il s'agit bien plus d'une question de faits que d'une question de droit. Néanmoins, on peut avancer que plus le pouvoir décisionnel d'une personne est grand, plus celle-ci est susceptible d'occuper une charge au sein de la municipalité. Toutefois, dans tous les cas, le fonctionnaire qui détient une charge a la capacité d'user de son influence pour empêcher ou favoriser l'adoption, par le conseil municipal, d'une ligne de conduite souhaitée ou d'une simple décision qu'il lui revient de prendre. Également, il peut s'agir pour le fonctionnaire municipal d'omettre d'accomplir un acte relevant de sa charge ou d'accomplir un acte donné.

Il semble que l'inspecteur municipal, l'urbaniste de la municipalité, le trésorier et les autres personnes occupant un poste semblable peuvent être considérés comme étant des fonctionnaires relevant d'un gouvernement municipal.

\section{Refus de remettre des biens (art. 337 du Code criminel)}

La peine maximale énoncée à l'article 337 du Code criminel est de quatorze ans, tandis que l'inhabilité prévue par la loi est de cinq ans. Cette disposition vise tant les fonctionnaires et employés municipaux que les membres 
du conseil municipal. En fait, elle concerne «quiconque, étant ou ayant été un employé au service [...] d'une municipalité, et chargé, en vertu de cet emploi, de la réception, de la garde, de la gestion ou du contrôle d'une chose, refuse ou omet de remettre cette chose à une personne qui est autorisée à la réclamer et qui, effectivement, la réclame».

Il n'est pas aisé de définir cette notion vu le manque de jugements traitant de celle-ci. Toutefois, nous croyons qu' un tel article vise sans doute les cas de vol ou les détournements à ses propres fins (outre la fraude) de biens, aussi large que puisse paraître cette expression, par un fonctionnaire, employé ou membre du conseil municipal d'une municipalité.

\section{Faux relevé de deniers publics par un fonctionnaire public (art. 399 du Code criminel)}

Suivant l'article 399 du Code criminel, toute personne qui est chargée de la réception, de la garde ou de la gestion de quelque partie des revenus publics et qui fournit sciemment un faux état ou un faux relevé, soit de deniers perçus par elle ou confiés à sa garde, soit de tout solde de deniers entre ses mains ou sous son contrôle, est coupable d'un acte criminel passible d'un emprisonnement maximal de cinq ans.

Bien entendu, cet article ne vise pas les cas d'erreur dans la préparation des états ou relevés, mais bien les cas où de tels documents sont falsifiés de façon intentionnelle par la personne en cause. En effet, outre le fait de présenter de faux documents, il faut avoir l'intention coupable de présenter ces documents avec les inscriptions fausses y figurant.

Puisque nous sommes en matière criminelle, il est juste d'appliquer au mot «sciemment» une portée autre que celle de nature civile que nous croyons applicable aux motifs d'inhabilité prévus à la Loi sur les élections et les référendums dans les municipalités. Ici, le mot «sciemment» revêt un caractère pénal, mettant en relief la volonté, la bonne ou la mauvaise foi du fonctionnaire ou employé dans l'exécution de ses tâches de préparation des documents précités. Aussi, celui-ci peut invoquer sa bonne foi à l'encontre de l'infraction reprochée pour tenter de s'exonérer des charges qui pèsent contre lui. 
Quant aux deniers publics, il s'agit simplement des argents faisant l'objet d'une gestion par le trésorier de la municipalité, le secrétaire-trésorier ou toute autre personne investie des pouvoirs de gérer un budget et de procéder à certains débours. Toutes ces opérations doivent inévitablement faire l'objet d'un compte rendu écrit au conseil municipal, tel compte rendu pouvant être présenté comme un état ou relevé des deniers sujets à sa gestion.

\section{D) LES POUVOIRS DU COMITÉ CONSULTATIF D'URBANISME}

\section{Le pouvoir d'étude et de recommandation}

Le C.C.U. étant créé par le conseil municipal d'une municipalité locale, ses pouvoirs ne peuvent, de toute évidence, outrepasser le cadre juridictionnel entourant et limitant les actes du conseil municipal. Aussi, l'expression «des pouvoirs d'étude et de recommandation en matière d'urbanisme, de zonage, de lotissement et de construction» prévue au paragraphe 2 de l'article 146 L.A.U. se voit d'emblée encadrée par les pouvoirs du conseil municipal en ces matières.

Le premier aspect de ce paragraphe, «soit les pouvoirs d'étude et de recommandation en matière d'urbanisme», couvre sans aucun doute tous les éléments pouvant être abordés dans le plan d'urbanisme de la municipalité. On se souviendra que celle-ci avait l'obligation d'adopter un plan d'urbanisme suivant l'entrée en vigueur du schéma d'aménagement de la municipalité régionale de comté sur le territoire de laquelle elle se situe ${ }^{21}$.

Le plan d'urbanisme est assujetti à un contenu obligatoire détaillé à l'article 83 L.A.U. :

Un plan d'urbanisme doit comprendre :

$1^{\circ}$ les grandes orientations d'aménagement du territoire de la municipalité;

$2^{\circ}$ les grandes affectations du sol et les densités de son occupation;

21. L.A.U., supra note 1 , art. 33 . 
$3^{\circ}$ le tracé projeté et le type des principales voies de circulation et des réseaux de transport.

Cet instrument de planification territoriale revêt un caractère relativement général, mais se doit tout de même de refléter le contenu du schéma d'aménagement. Il en va de même pour le contenu facultatif du plan d'urbanisme prévu à l'article $84 L . A . U .^{22}$, ainsi qu'à l'égard des programmes particuliers d'urbanisme pouvant être intégrés au plan d'urbanisme et dont le contenu se doit de respecter les articles 85 et 85.1 L.A.U. ${ }^{23}$ Dans l'exercice de

22. L.A.U., supra note 1, art. 84 : 84. Un plan d'urbanisme peut comprendre :

$1^{\circ}$ les zones à rénover, à restaurer ou à protéger;

$2^{\circ}$ (paragraphe supprimé) [L.Q. 1993, c.3, a.44];

$3^{\circ}$ la nature, la localisation et le type des équipements et infrastructures destinés à l'usage de la vie communautaire;

$4^{\circ}$ les coûts approximatifs afférents à la réalisation des éléments du plan;

$5^{\circ}$ la nature et l'emplacement projeté des principaux réseaux et terminaux d'aqueduc, d'égouts, d'électricité, de gaz, de télécommunications et de câblodistribution;

$\sigma^{\circ}$ la délimitation à l'intérieur du territoire municipal d'aires d'aménagement pouvant faire l'objet de programmes particuliers d'urbanisme;

$7^{\circ}$ la délimitation à l'intérieur du territoire municipal d'aires d'aménagement pouvait faire l'objet de plans d'aménagement d'ensemble conformément aux articles 145.9 à 145.14.

23. Ibid., art. 85 : 85 Un plan d'urbanisme peut aussi comprendre un programme particulier d'urbanisme pour une partie du territoire de la municipalité.

Ce programme d'urbanisme peut comprendre :

$1^{\circ}$ l'affectation détaillée du sol et la densité de son occupation;

$2^{\circ}$ le tracé projeté et le type des voies de circulation, des réseaux de transport, d'électricité, de gaz, de télécommunications et de câblo-distribution:

$3^{\circ}$ la nature, l'emplacement et le type des équipements et des infrastructures destinés à l'usage de la vie communautaire;

$4^{\circ}$ la nomenclature des travaux prévus, leurs coûts approximatifs de réalisation et une indication des organismes concernés;

$5^{\circ}$ les règles de zonage, de lotissement et de construction proposées;

$6^{\circ}$ la séquence de construction des équipements urbains et des réseaux et terminaux d'aqueduc et d'égouts;

$7^{\circ}$ la durée approximative des travaux;

$8^{\circ}$ les programmes particuliers de réaménagement, de restauration et de démolition.

Un programme particulier d'urbanisme applicable à la partie du territoire de la municipalité désignée comme son «centre-ville» ou son «secteur central» peut aussi comprendre un programme d'acquisition d'immeubles en vue de leur aliénation ou de leur location à des fins prévues dans le programme particulier d'urbanisme.

Ibid. art. 85-1 : 85.1 Une municipalité peut adopter un programme particulier d'urbanisme pour la partie de son territoire qu'elle désigne comme son «centre-ville» ou son «secteur central», sans que ce programme fasse partie de son plan d'urbanisme, tant qu'elle n'a pas 
leur pouvoir d'étude et de recommandation en matière d'urbanisme, il est indispensable que les membres du C.C.U. connaissent le contenu du schéma d'aménagement de manière à bien orienter le conseil municipal dans sa prise de décision au niveau local.

C'est justement à ce niveau que le C.C.U. intervient le plus souvent puisque le conseil municipal a l'obligation d'adopter, suivant l'entrée en vigueur du plan d'urbanisme ${ }^{24}$, des règlements dits à «caractère normatif» que sont le règlement de zonage $^{25}$, le règlement de lotissement ${ }^{26}$, le règlement de construction $^{27}$ ainsi que le règlement sur les conditions de délivrance d'un permis de construction ${ }^{28}$ dans le cas où le document complémentaire au schéma d'aménagement l'exige ${ }^{29}$. Cette nécessité découle du fait que le plan d'urbanisme n'a pas d'effet juridique sur les citoyens ${ }^{30}$ et qu'une précision documentaire accrue s'avère péremptoire pour que le pouvoir ministériel des fonctionnaires municipaux soit valablement exercé ${ }^{31}$. Aussi, le C.C.U. n'est

adopté ce plan et tant qu'un schéma d'aménagement n'est pas en vigueur dans son territoire. Une municipalité qui adopte un programme prévoyant plusieurs «centre-villes» ou «secteurs centraux»sur son territoire peut prévoir des règles différentes à l'égard de chacun d'eux.

Les dispositions de la présente loi relatives au plan d'urbanisme s'appliquent à ce programme, compte tenu des changements nécessaires, sauf les articles 83, 84 et 98.

En plus des éléments mentionnés à l'article 85, ce programme doit comprendre les grandes orientations d'aménagement du territoire de la municipalité qui concernent la partie de ce territoire à laquelle il s'applique.

Dans le cas d'une municipalité dont le territoire est compris dans celui d'une municipalité régionale de comté qui a commencé l'élaboration de son premier schéma, ce programme entre en vigueur à la date de son approbation par le conseil de la municipalité régionale de comté. Dans les autres cas, il entre en vigueur à la date de la publication du règlement par lequel il est adopté, conformément à la loi qui régit la municipalité, ou à la date ultérieure qui y est prévue.

24. Ibid., art. 102.

25. Ibid., art. 113.

26. Ibid., art. 115 .

27. Ibid., art. 118.

28. Ibid., art. 116.

29. Ibid., art. 102.

30. Lynch c. Aylmer (City of), [1989] R.D.I. 768 (C.S.); Cousineau c. Boucherville (Ville de), [1986] R.J.Q. 318 (C.S.) [ci-après Cousineau].

31. Descheneaux c. Saint-Jean-Baptiste-de-Nicolet (Corporation municipale de), J.E. 82-1094 (C.S.); Estérel (Ville d') c. Grundman, [1983] R.L. 451 (C.S.); Rouyn-Noranda (Ville de) c. Girard, J.E. 88-1004 (C.S.); 146726 Canada Inc. (Sparta Café) c. Montréal (Ville de) (10 
aucunement responsable de l'émission des permis et l'exercice d'un tel pouvoir constitue sans aucun doute une sous-délégation illégale de pouvoir ${ }^{32}$.

L'exercice d'un pouvoir d'étude et de recommandation demeure donc entièrement assujetti à la volonté du conseil municipal et ne constitue pas une sous-délégation illégale de pouvoir. Ce fut d'ailleurs l'orientation retenue par la Cour supérieure dans l'affaire Mandel c. Verdun (Ville de) ${ }^{33}$ où la Cour entérina le procédé par lequel la municipalité procéda à une adoption de principe d'un règlement, le soumit pour étude à ses consultants, puis adopta le rapport par résolution de manière à assujettir toute demande ultérieure de permis au respect de ces normes. La Cour jugea que les consultants n'avaient, en réalité, qu'un pouvoir de recommandation de nature à éclairer les décisions du conseil municipal et qu'en agissant ainsi, ce dernier ne confiait pas à un tiers le soin de légiférer à sa place.

\section{Le rôle du C.C.U. dans l'application des règlements à caractère discrétionnaire}

Le paragraphe 2 de l'article 146 L.A.U. couvre certainement, bien qu'indirectement, une autre réalité. En effet, l'existence d'un C.C.U. s'avère une condition préalable essentielle à l'application des règlements dits à «caractère discrétionnaire» que sont le règlement sur les dérogations mineures $^{34}$, le règlement sur les plans d'aménagement d'ensemble ${ }^{35}$ et le règlement sur les plans d'implantation et d'intégration architecturale ${ }^{36}$. Bien que le règlement sur les ententes relatives aux travaux municipaux ${ }^{37}$ puisse également être considéré, dans une moindre mesure, comme discrétionnaire, il n'implique toutefois pas de participation directe du C.C.U.

juillet 1996), Montréal 500-05-012530-950 (C.S.)

32. Prime c. Saint-Lambert (Town of), [1974] C.S. 536.

33. (25 avril 1986), Montréal 500-05-002712-865 (C.S.).

34. L.A.U., supra note 1 , art. 145.1 et s.

35. Ibid., art. 145.9 et s.

36. Ibid., art. 145.15 et s.

37. Ibid., art. 145.21 et s. 
Le principe à la base de l'existence des règlements à caractère discrétionnaire est à l'effet d'attribuer au conseil municipal le pouvoir de se prononcer, par résolution, sur chaque projet qui lui est soumis en application des règlements précités. Cette décision doit, pour être valide, se voir préalablement enrichie de l'opinion du C.C.U.

Ainsi, dans le cadre du règlement sur les dérogations mineures, l'article 145.7 L.A.U. prévoit que :

145.7 Le conseil rend sa décision après avoir reçu l'avis du comité consultatif d'urbanisme.

Une copie de la résolution par laquelle le conseil rend sa décision doit être transmise à la personne qui a demandé la dérogation.

Pour sa part, l'article 145.12 L.A.U. relatif à l'approbation des plans d'aménagement d'ensemble prévoit que :

145.12 Le conseil d'une municipalité qui a adopté un règlement visé à l'article 145.9 doit, après consultation du comité consultatif d'urbanisme, approuver ou refuser par résolution un plan d'aménagement d'ensemble qui lui est présenté conformément à la présente section.

Une copie de cette résolution doit être transmise à la personne qui a présenté ce plan.

Quant aux plans d'implantation et d'intégration architecturale, c'est l'article 145.19 L.A.U. qui prévoit que :

145.19 À la suite de la consultation du comité consultatif d'urbanisme et, le cas échéant, de celle décrétée en vertu de l'article 145.18, le conseil de la municipalité approuve les plans s'ils sont conformes au règlement ou les désapprouve dans le cas contraire.

La résolution désapprouvant les plans doit être motivée. 
Soulignons en terminant que depuis 1985 , le C.C.U. a vu son pouvoir de recommandation étendu à l'application de certains articles de la Loi sur les biens culturels $^{38}$. Suivant les articles 59 et suivants de cette loi, le C.C.U. peut, entre autres, donner son avis à la demande du conseil municipal sur toute question relative à la protection des biens culturels par la municipalitée ${ }^{39}$. Il pourra également entendre les personnes sur toute question relevant de sa compétence $^{40}$ et la municipalité devra prendre son avis avant de citer un monument historique dans l'intérêt public ${ }^{41}$. Il interviendra également une fois le règlement de citation adopté ${ }^{42}$ ou lorsque l'on voudra démolir, déplacer ou utiliser le monument historique ${ }^{43}$ ou lorsque la municipalité désirera prendre des mesures pour mettre en valeur le voisinage du monument historique ${ }^{44}$.

\section{E) LES RÈGLES DE RÉGIE INTERNE}

Le troisième paragraphe de l'article 146 L.A.U. laisse au C.C.U. le soin d'adopter ses propres règles de régie interne. À cet égard, le C.C.U. pourra s'inspirer des règles qui régissent la conduite du conseil municipal pour déterminer, notamment, la fréquence de ses réunions, le mode de convocation de ses réunions, ordinaires ou spéciales, le quorum requis pour la tenue d'une réunion, les modalités entourant le vote de ses membres, le vote prépondérant du président ainsi que la tenue des procès-verbaux par le secrétaire. Eu égard à ce dernier point, il est bon de rappeler la décision de la Commission d'accès à l'information dans l'affaire Desrochers c. Pointe-Claire (Ville de) ${ }^{45}$ à l'effet que les procès-verbaux du C.C.U., dans la mesure où ils font l'objet de délibérations de la part du conseil municipal, sont accessibles au public, sous réserve de l'article 38 de la Loi sur l'accès aux documents des organismes

\footnotetext{
38. L.R.Q. c. B-4 [ci-après L.B.C.]; voir la décision dans l'affaire Bera Enterprises Ltd. c. Vallée, [1988] R.D.I. 4 (C.S.).

$39 . \quad$ L.B.C., ibid., art. 61.

40. Ibid., art. 62 (2).

41. Ibid., art. 70 .

42. Ibid., art. 80 .

43. Ibid., art. 81-82.

44. Ibid., art. 87.

45. [1984-86] 1 C.A.I. 293.
} 
publics et sur la protection des renseignements personnels ${ }^{46}$, lequel se lit comme suit :

Un organisme public peut refuser de communiquer un avis ou une recommandation que lui a fait un organisme qui en relève ou qu'il a fait à un autre organisme public, jusqu'à ce que la décision finale sur la matière faisant l'objet de l'avis ou de la recommandation ait été rendue publique par l'autorité compétente.

Il en est de même pour un ministre en ce qui concerne un avis ou une recommandation que lui a fait un organisme qui relève de son autorité.

Dans le contexte actuel où la jurisprudence se fait de plus en plus sévère en matière de conflits d'intérêts, il n'est certes pas superflu d'envisager l'adoption d'un code d'éthique destiné à guider les membres du C.C.U. lors de la prise en considération de certaines questions. À cet effet, les motifs d'inhabilité prévus à la Loi sur les élections et les référendums dans les municipalités constituent un guide qui nous apparaît adéquat pour régir les comportements à adopter lors de situations soulevant un doute quant à l'impartialité d'un membre du C.C.U. ${ }^{47}$

Enfin, les membres du C.C.U. doivent conserver à l'esprit que les plans d'architecte ou d'ingénieur qui lui sont soumis dans le cadre du règlement sur les plans d'aménagement d'ensemble, du règlement sur les plans d'implantation ou d'intégration architecturale ou dans le cadre du règlement sur les dérogations mineures ne sont pas, suivant une jurisprudence majoritaire de la Commission d'accès à l'information ${ }^{48}$, accessibles au public et qu'ils sont, à toutes fins pratiques, confidentiels. Cette jurisprudence est à l'effet que ces documents tombent sous le coup de l'exception au principe de l'accessibilité aux documents

46. L.R.Q. c. A-2.1 [ci-après L.A.I.].

47. Voir Gale, supra note 16.

48. $\quad$ Ferahian c. Westmount (Ville de), [1986] C.A.I. 166; Juneau c. Québec (Ville de), [1989]

C.A.I. 245. 
Loi sur l'aménagement et l'urbanisme

prévue à l'article 9 L.A.I., qu'il y ait ou non dépôt de ces derniers aux archives de la municipalitét ${ }^{4}$.

\section{SECTION II- LE RÈGLEMENT SUR LES DÉROGATIONS MINEURES}

\section{A) LE CHAMP D'APPLICATION}

La réglementation d'urbanisme des municipalités et plus particulièrement ses règlements de zonage et de lotissement, doivent prévoir des normes strictes et objectives qui encadrent le fonctionnaire municipal dans l'exercice de son pouvoir ministériel d'application de cette réglementation. À cet égard, la Cour supérieure écrivant dans l'affaire Estérel (Ville d') c. Grundman $^{50}$ :

Un règlement doit être objectif et stipuler des normes, des critères et des directives suffisamment déterminées, suffisamment précises pour que non seulement le citoyen sache et connaisse les formalités auxquelles il doit se soumettre préalablement à l'obtention d'un permis mais, encore, pour que la corporation municipale, par la suite, puisse rendre applicable son règlement, étant consciente que les impératifs qu'elle prescrit sont connus de ses citoyens et que l'application peut être objective. De là découle évidemment que le règlement doit être rédigé dans une forme qui laisse connaître les exigences précises de la corporation municipale et non pas rapporter des termes vagues, laissant le champ libre à toutes sortes d'interprétations et de spéculations qui rendent l'administration d'une ville, à toutes fins utiles, incohérente. archives municipales», [1995] 2 B.D.M. 62-64.

50. [1983] R.L. 451 (C.S.) à la p. 455; voir aussi : Lambert-Bédard c. Sillery (Ville de), J.E. 80630 (C.S.); Montréal (Ville de) c. Arcade Amusements Inc. (1985), 1 R.C.S. 368; 110806 Canada Inc. c. Carignan (Ville de), J.E. 85-672 (C.A.); Didus c. Wentworth-Nord (Municipalité de), J.E. 93-65 (C.S.). 
Du fait de cette «rigidité», la réglementation d'urbanisme ne laisse guère place aux cas d'exception qui nécessitent un traitement particulier. Il n'est pas rare qu'un règlement de zonage, de par la rigueur des normes qui y sont prévues, frustre un constructeur ou un résident dont le projet déroge, par exemple, aux marges de recul minimales ou aux différents coefficients liés à l'implantation d'un bâtiment prévus au règlement de zonage.

La L.A.U. prévoit une procédure de changement de zonage longue et complexe. Outre les frais qui doivent être payés à la municipalité par les initiateurs d'une telle démarche et qui sont, le plus souvent, considérables, celuici devra s'armer de patience et assister à l'adoption par le conseil municipal d'un premier projet de règlement modificateur ${ }^{51}$, à la publication d'un avis public annonçant la tenue d'une assemblée de consultation publique sur ce projet de règlement ${ }^{52}$, à la tenue de cette assemblée de consultation ${ }^{53}$, à l'adoption d'un second projet de règlement modificateur ${ }^{54}$, à la publication d'un avis public destiné cette fois à isoler les dispositions du règlement pouvant faire l'objet d'une demande d'approbation ${ }^{55}$ puis, si telle demande est déposée à la municipalité, à souffrir la tenue d'un registre et, possiblement, celle d'un référendum ${ }^{56}$. La procédure est en effet la même qu'il s'agisse d'une demande relative à la réduction d'une marge de recul avant sur un lot résidentiel que d'une modification à la classification des usages pour une zone entière. Le caractère fastidieux de cette procédure ainsi que le risque latent qu'une modification du règlement de zonage soit perçue comme étant du rezonage

51. L.A.U., supra note 1 , art. 124.

52. Ibid., art. 126.

53. Ibid., art. 125, 127.

54. Ibid., art. 128.

55. Ibid., art. 132.

56. Ibid., art. 136.1 et Loi sur les élections et les référendums dans les municipalités, supra note 15 , art. 518-579. 
parcellaire (spot zoning) $)^{57}$ témoignent du peu d'intérêt que suscite normalement une telle démarche.

Il existe toutefois un «remède» à cette procédure, lequel fut introduit par le législateur en 1985 lors de l'adoption de la Loi modifiant diverses dispositions législatives concernant les municipalités ${ }^{58}$ et l'inclusion à la L.A.U. des articles

57. Voir la jurisprudence suivante pour l'application de la théorie du «spot zoning»: Scarborough (Township of) c. Bondi, [1959] R.C.S. 444; Mounterbrooke v. Montréal (City of), [1963] R.L. 28 (C.S.); Intertrade Industries Ltd. c. Côte Saint-Luc (Cité de), [1965] C.S. 369; Sula c. Duvernay (Cité de), [1970] C.A. 234; Ivanhoe Corporation c. Val-d'Or (Ville de), [1973] C.S. 904; Lind c. Beaconsfield (City of), [1976] C.S. 1065; Entreprises Herskel Ltd. c. Greenfield Park (Town of), [1977] C.S. 396; Lamoureux c. Beaconsfield (Ville de), [1978] 1 R.C.S. 134; Ruscito c. Montréal-Est (Ville de) (20 janvier 1978), Montréal 500-05022443-772 (C.S.); Bider c. Baie-d'Urfé (Town of) (9 mai 1980), Montréal 500-05-008365767 (C.S.); Aubé c. Loretteville (Ville de), [1981] J.M. 132 (C.S.); Desrosiers c. Longueuil (Ville de), J.E. 81-593 (C.S.); Grantham-Ouest (Corporation municipale de) c. Deblois, J.E. 82-1093 (C.S.); Cousineau, supra note 30 ; Rocheleau c. Repentigny (Ville de), [1986] R.J.Q. 439 (C.S.); Juneau c. Québec (Ville de) (1 $1^{\mathrm{er}}$ juin 1987), Québec 200-05-002019-870 (C.S.), conf. par [1991] R.J.Q. 2781 (C.A.); Lafond c. Saint-Hyacinthe (Ville de) (5 octobre 1988), Saint-Hyacinthe 750-05-00262-872 (C.S.); Browns c. Sainte-Adèle (Corporation municipale de) (31 mai 1988), Terrebonne 700-05-001259-872 (C.S.); Hôpital CharlesLemoyne c. Greenfield Park (Ville de), J.E. 90-203 (C.S.); Bellington c. Aylmer (Ville d'), [1991] R.J.Q. 1283 (C.S.); Sable des Forges Inc. c. Trois-Rivières (Ville de), J.E. 91-1760 (C.S.); Carrières Rive-Sud Inc. c. Boucherville (Ville de), [1993] R.J.Q. 130 (C.S.), conf. par J.E. 99-629 (C.A.); Gagné c. Lac-Simon (Corporation municipale du), [1994] R.J.Q. 453 (C.S.); Decelles c. Lac-Brome (Corporation municipale de la ville de), L.P.J. 94-1993 (C.S.); Saint-Rémi (Ville de) c. Centre communautaire Saint-Rémi Inc., J.E. 95-247 (C.S.); Léonard c. Bonaventure (Municipalité de), J.E. $98-452$ (C.S.); Basil Holding Corp. c. Côte Saint-Luc (Cité de), J.E. 98-960 (C.S.); Veilleux c. Pièces d'autos Roch Drouin Inc., J.E. 99921 (C.A.) confirmant J.E. 96-341 (C.S.); Propriétés immobilières Oshawa Inc. c. Laval (Ville de), J.E. 98-1302 (C.S.).

58. Loi modifiant diverses dispositions législatives concernant les municipalités, L.Q. 1985, c.27, art. 169; G.O.Q. 1985.II.4233; voir à cet effet : P. Kenniff, «Approche réglementaire de l'aménagement urbain» (1977), 18 C. de D. 797; B. Beaulieu, Y. Ferland et F. Roy, L'arpenteur-géomètre et les pouvoirs municipaux en aménagement du territoire et en urbanisme, Cowansville (Qc), Yvon Blais, 1995 aux pp. 253-259; Y. Duplessis et J. Hétu, «La Loi sur l'aménagement et l'urbanisme» dans Chambre des notaires du Québec, Répertoire de droit, Montréal, 1991 aux pp. 218-240; L. Giroux, «Quelques problèmes d'actualité en droit de l'aménagement - Les normes d'implantation, la hauteur, l'accès au terrain, les clôtures, l'apparence extérieure et le stationnement, la dérogation mineure, les droits acquis et le lotissement» (1988), 5 C.P. du N. 131; J. L'Heureux, «Le CCU et les dérogations mineures - l'appréciation des demandes» (1993), 13/2 R.Q.U. 11; «Les dérogations à l'interdiction de construire en zone inondable», Muni-Express, ${ }^{\circ}$ 05, 11 mai 
145.1 à 145.8. Depuis le 20 juin 1985, «le conseil d'une municipalité dotée d'un comité consultatif d'urbanisme peut adopter un règlement sur les dérogations mineures aux dispositions des règlements de zonage et de lotissement autres que celles qui sont relatives à l'usage et à la densité d'occupation du sol ${ }^{59}$. C'est le conseil municipal qui décidera donc, par résolution, d'accorder une telle dérogation sans devoir préalablement procéder à une modification de sa réglementation ${ }^{60}$. En elle-même, la résolution constitue une sorte de «mini» règlement de zonage attaché à l'immeuble qu'elle touche sans pour autant constituer un permis ou certificat. Ainsi, c'est sur présentation de la résolution accordant une dérogation mineure et sur paiement du tarif requis que le fonctionnaire responsable délivrera le permis ou certificat requis, conformément à l'article 145.8 L.A.U.

Bien que la portée de l'article 145.1 L.A.U. soit claire eu égard à l'impossibilité d'accorder une dérogation mineure en matière d' "usage», elle est toutefois moins bien définie lorsqu'il s'agit de l'appliquer à l'expression «densité d'occupation du sol». Le concept de «densité d'occupation du sol» énoncé à l'article 113, alinéa 2 paragraphe 3 L.A.U. demeure, encore de nos jours, une abstraction peu traitée par la doctrine et la jurisprudence. Aussi, il est plus facile de déterminer le contenu de ce concept par exclusion que par inclusion puisque celui-ci n'est pas défini à la L.A.U., tout comme la notion semblable de «densité approximative d'occupation ${ }^{61}$. D'abord, il y a lieu de le situer dans le cadre d'application de cette loi à l'intérieur de l'article 113 alinéa 2 paragraphe 3 qui se libelle ainsi :

1999; Ministère des Affaires municipales, Direction générale de l'urbanisme et de l'aménagement du territoire, CCU-4-Les dérogations mineures, 1991, Québec; CCU -5- Les dérogations mineures - Démarche d'évaluation des demandes, 1991, Québec; Ministère des Affaires municipales, Direction générale de l'urbanisme et de l'aménagement du territoire, Les dérogations mineures aux règlements d'urbanisme, Aménagement et Urbanisme, 1986, Québec.

59. L.A.U., supra note 1 , art. 145.1.

60. Voir relativement aux normes en vigueur dans le reste du Canada : I. Rogers, Canadian Law of Planning and Zoning, Toronto, Carswell, mise à jour aux pp. 209-210.

61. L.A.U., supra note 1 , art. 6 (1). 
Spécifier, pour chaque zone, les constructions ou les usages qui sont autorisés et ceux qui sont prohibés, y compris les usages et édifices publics, ainsi que les densités d'occupation du sol.

La notion de «densité d'occupation du sol» s'occupe à circonscrire, de manière relativement générale, la question du rapport entre la superficie des constructions implantées sur un terrain et celle du terrain en tant que tel de manière à harmoniser la densité de construction ${ }^{62}$.

La Cour supérieure a ainsi défini la «densité d'occupation du sol» dans l'affaire Sillery (Ville de) c. Villa Ignatia Inc. ${ }^{63}$ :

On entend par densité d'occupation du sol, le nombre total de logements compris à l'intérieur d'un périmètre. Il est possible de déterminer la «densité brute», c'est-à-dire le nombre total de logements compris à l'intérieur d'un périmètre divisé par le nombre total d'hectares visés, incluant les rues et tout terrain affecté à un usage public ou institutionnel à même ce périmètre, et la «densité nette», c'est-à-dire le nombre de logements compris ou prévus sur un hectare de terrain à bâtir affecté spécifiquement à l'habitation, excluant toute rue publique ou privée ainsi que tout terrain affecté à un usage public ou institutionnel.

Suivant cette définition du concept de «densité d'occupation du sol» que la Cour semble, par ailleurs, rattacher à un usage strictement résidentiel, on comprend que la «densité d'occupation $d u$ sol» est un concept global qui fait référence à l'utilisation du territoire sur la base de l'implantation de constructions au sol et de l'espace occupé par celles-ci. Dans chacun des cas, la qualification qualitative de l'habitation permise (unifamiliale, multifamiliale ...) est essentielle pour assurer une certaine cohérence à l'intérieur de la zone quant aux orientations du schéma ou du plan d'urbanisme reliées à la densité

62. L. Giroux et M. Poirier, «Les notions d'usage et de densité d'occupation du sol, de même que les problèmes relatifs aux avis de motion dans la Loi sur l'aménagement et l'urbanisme : des questions toujours d'actualité» dans Service de la formation permanente, Barreau du Québec, Développements récents en droit municipal (1998), Cowansville (Qc), Yvon Blais, 1998, 195.

63. J.E. 91-1416 (C.S.). 
globale autorisée, cette qualification présentant la densitée préconisée dans la zone. De prime abord, la définition de la Cour supérieure nous amène à croire que les rapports quantitatifs que sont le nombre de logements par bâtiment ou par hectare sont les seuls pouvant être considérés dans la définition de la «densité d'occupation du sol». Toutefois, il va sans dire qu'à la base, le concept de «densité d'occupation du sol» en urbanisme est un concept très large qui fait référence à un nombre considérable de «rapports quantitatifs», que l'on parle seulement des coefficients d'emprise au sol, des coefficients d'occupation du sol, de la couverture du lot (exprime le C.E.S. en \%), de l'espace de référence linéaire ou de la superficie ou de la dimension des lots. En fait, plusieurs des normes d'implantation édictées dans un règlement de zonage, comme le nombre d'étages par exemple, ont un impact direct sur la densité.

On peut donc dire que le concept général de «densité d'occupation du sol» est composé de plusieurs rapports quantitatifs tous élaborés en fonction des superficies autorisées des bâtiments et des terrains concernés.

Pour leur part, les articles 145.1 et suivants L.A.U. relatifs aux pouvoirs des conseils municipaux en matière de dérogations mineures excluent la possibilité d'accorder de telles dérogations pour des questions de «densité d'occupation du sol». À ce stade, nous sommes d'opinion que le texte de la loi peut être lu en gardant à l'esprit la définition de «densité d'occupation du sol» donnée par la Cour dans l'affaire Sillery (Ville de) c. Ville Ignatia Inc. ${ }^{64}$ et que les articles précités réfèrent, implicitement du moins, à l'article 113 alinéa 2 paragraphe 3 L.A.U. En conséquence, les rapports découlant d'un calcul du nombre de logements par bâtiment et du nombre de logements par hectare (densité brute ou nette) ne pourraient être «modifiés» par l'octroi d'une dérogation mineure.

Cependant, tous les rapports précités, comme les coefficients d'emprise au sol, les coefficients d'occupation du sol, la couverture du lot, l'espace de référence linéaire, la superficie ou la dimension des lots, expriment eux aussi des

64. Voir aussi les affaires Michaud-Matte c. Sillery (Ville de), J.E. 85-128 (C.S.) et Société immobilière Manitonga Soutana c. Mont-Tremblant (Corporation municipale de), J.E. 92977 (C.S.). 
rapports de «densité» sous le couvert toutefois des articles 113, alinéa 2 paragraphe 5 et 6 et 115, alinéa 2 paragraphes 1 à 4 . Comme le législateur n'exclut aux articles 145.1 et suivants L.A.U. l'octroi de dérogations mineures que pour des questions "d'usage ou de densité d'occupation du sol», il nous apparaît logique de prétendre que ce dernier désirait référer à la «densité d'occupation $d u$ sol» prévue à l'article 113, alinéa 2 paragraphe 3 L.A.U. En l'espèce, il appert forcément que tous les autres rapports de «densité» édictés sous l'empire des articles précités se distinguent du concept de «densité d'occupation $d u$ sol» dans le traitement des dérogations mineures et qu'en ce sens, ils ne semblent pas sujets à l'exclusion prévue aux articles 145.1 et suivants L.A.U.

Mais que signifie donc l'expression «densité d'occupation du sol» du paragraphe 3 des articles 113 et 145.1 et suivants L.A.U. s'il faut en soustraire la substance des paragraphes 5 et 6 de l'article 113 ainsi que celle des paragraphes 1 à 4 de l'article 115? Doit-on limiter son contenu à la définition qu'en donne la Cour supérieure dans l'affaire Villa Ignatia et considérer qu'il s'agit d'une notion d'application purement résidentielle? Si tel est le cas, c'est oublier que tous les rapports de «densité», que l'on parle des différents coefficients ou des mesures de superficie des planchers, des bâtiments ou des lots ou que l'on parle des différentes normes d'implantation des bâtiments, ont tous un impact direct sur la densité d'occupation du sol et que l'octroi de dérogations mineures à l'égard de telles normes semble aller à l'encontre de la volonté même du législateur. En conséquence, si le concept de «densité d'occupation du sol» en droit de l'urbanisme apparaît nécessairement contenir une qualification qualitative (unifamiliale ...) des bâtiments ainsi que différents rapports liés à la conception quantitative (C.O.S., C.E.S,....) de l'aménagement du territoire, qu'en est-il de ce concept au sens de la L.A.U. si tout son contenu semble avoir été disloqué en autant d'articles distincts qui, faut-il le rappeler, ne sont pas des sous-rubriques de l'article 113, alinéa 2 paragraphe 3 L.A.U.? Enfin, une telle réduction de la portée de l'expression «densité d'occupation du sol» nous apparaît quelque peu artificielle, bien que conforme au texte de la loi 
et contraire à la définition retenue par le ministère des Affaires municipales dans un guide de présentation $^{65}$ :

Densité d'occupation du sol : La notion de densité du sol se rapporte à une répartition d'éléments (structure, construction, logement) ou d'habitants par unité de surface. En matière d'urbanisme et d'aménagement du territoire, les éléments suivants sont habituellement mis en rapport avec une superficie donnée de territoire :
- $\quad$ la superficie d'un bâtiment au sol;
- $\quad$ la superficie totale de plancher d'un bâtiment par rapport à la superficie totale du lot;
- $\quad$ le nombre de logements;

Les valeurs de densité sont approximatives quand elles s'expriment par des moyennes ou encore lorsqu'elles fixent des minima et maxima ou l'un ou l'autre.

\section{B) L'ADOPTION ET LE CONTENU DU RÈGLEMENT SUR LES DÉROGATIONS MINEURES}

L'adoption par le conseil municipal d'un règlement sur les dérogations mineures, est assujettie à la procédure de consultation publique prévue aux articles 123 et suivants L.A.U. et à la constitution préalable d'un C.C.U. Conformément à l'article 145.3 L.A.U., le règlement sur les dérogations mineures doit contenir trois volets particuliers, ce qui ne nous apparaît pas pour autant limiter le pouvoir du conseil municipal d'adopter des règles supplémentaires.

En premier lieu, le règlement doit prévoir «la procédure requise pour demander au conseil d'accorder une dérogation et les frais exigibles pour l'étude de la demande» ${ }^{66}$. Outre la rémunération des membres du C.C.U., s'ils sont rémunérés, les frais pourront comprendre les frais de publication de l'avis

65. Ministère des Affaires municipales, Direction générale de l'urbanisme et de l'aménagement du territoire, Répertoire commenté des termes, des organismes et des lois, Québec, Direction générale des publications gouvernementales, 1985 à la p. 42.

66. L.A.U., supra note 1, art. 145.3 (1). 
public exigé par l'article 145.6 L.A.U. relatif à la discussion devant être tenue par le conseil municipal sur la nature et les effets de la dérogation demandée. Quant à la procédure, d'ordinaire fort simple, elle devra prévoir que le conseil municipal se prononcera lors d'une séance publique sur la demande de dérogation mineure après avoir reçu l'avis du C.C.U. et entendu tout intéressé relativement à cette demande.

L'avis public doit être publié au moins 15 jours avant la tenue de la séance à laquelle le conseil municipal doit statuer sur la demande de dérogation mineure. Il appert toutefois de la décision de la Cour supérieure dans l'affaire Lis c. Kemeny ${ }^{67}$ que «même si le délai entre la publication dans les journaux et la tenue de la séance du conseil n'est que de douze jours, l'article 23 C.M.Q. couvrirait cette informalité [si] aucune injustice réelle n'a été prouvée». Il faut noter cependant que la procédure de publication de l'avis public est différente selon que la municipalité est régie par la L.C.V. ou par le C.M.Q. Dans le premier cas, l'avis doit être publié dans un journal français circulant dans la municipalité et affiché au bureau de la municipalitée ${ }^{68}$, alors que, dans le second cas, la publication se fait en affichant une copie de cet avis dans la municipalité à deux endroits différents ${ }^{69}$.

En second lieu, le règlement devra identifier, «parmi les zones prévues par le règlement de zonage, [...] celles où une dérogation mineure peut être accordée ${ }^{70}$. L'article 145.3 (2) L.A.U. prévoit spécifiquement que ce sont «les zones prévues par le règlement de zonage» qui peuvent servir de division territoriale aux fins du règlement sur les dérogations mineures [nous soulignons]. Cela nous renvoit de toute évidence au paragraphe $1 \mathrm{du}$ second alinéa de l'article 113 L.A.U. qui prévoit que la municipalité peut adopter un plan de zonage qui divise le territoire de la municipalité en zones. En conséquence, il n'est pas indispensable ni même nécessaire que le règlement sur les dérogations mineures porte sur l'ensemble du territoire de la municipalité.

67. J.E. 96-2013 à la p. 20.

68. L.C.V., supra note 7 , art. $345,361$.

69. C.M.Q., supra note 8, art 450.

70. L.A.U., supra note 1 , art. 145.3 (2). 
En troisième lieu, le règlement devra énumérer «les dispositions des règlements de zonage et de lotissement qui peuvent faire l'objet d'une dérogation mineure ${ }^{71}$. Ce paragraphe doit se lire en tenant compte des limites intrinsèques de ce pouvoir prévues à l'article 145.1 L.A.U. eu égard à l'impossibilité d'accorder des dérogations mineures en ce qui a trait aux dispositions des règlements de zonage et de lotissement qui sont relatives à l'usage et à la densité d'occupation du sol. Mentionnons, enfin, que «toutes les dérogations mineures doivent être énumérées dans le règlement, autrement le conseil n'a pas le pouvoir de les accorder» ${ }^{72}$.

\section{C) L'ÉPOQUE DE LA PRÉSENTATION DE LA DEMANDE}

La demande de dérogation mineure pourra intervenir tant au début du processus devant mener à l'érection d'une construction qu'à la toute fin des travaux lorsqu'une contravention à la réglementation d'urbanisme est constatée. Ainsi, une demande peut être formulée au moment d'une demande de permis ou de certificat s'il est impossible de modifier le projet pour le rendre conforme. Elle peut également l'être pendant la réalisation des travaux si la modification de la construction s'avère un remède disproportionné par rapport à la dérogation reprochée. Enfin, elle pourra également être formulée dans l'objectif de régulariser la situation d'un immeuble dérogeant au règlement de zonage ou de lotissement de la municipalité.

Dans tous les cas, l'article 145.5 L.A.U. exige que les travaux en cause aient préalablement fait l'objet d'un permis d'un construction et qu'ils aient également été effectués de bonne foi. Compte tenu qu'il est difficile de prétendre à la bonne foi en cas d'absence d'un permis de construction, le législateur en a fait des conditions cumulatives. À tout événement, la détermination de la bonne foi demeure essentiellement une question de faits soumise à l'appréciation de la $\mathrm{Cour}^{73}$.

71. Ibid., art. 145.3 (3).

72. 151951 Canada Inc. c. Val-des-Monts (Municipalité de), [1990] R.J.Q. 834 (C.S.).

73. Auger c. Lévis (Ville de) (4 mai 1989), Québec 200-05-000899-977 (C.S.); Saint-AndréAvellin (Corporation municipale de la paroisse de) c. Bertrand-Varin (6 mai 1996), Hull 550-05-000581-952 (C.S.) [ci-après Saint-André-Avellin (Corporation municipale de la paroisse de)]. 
Enfin, il est intéressant de constater que l'alternative de la dérogation mineure pourra être invoquée à l'encontre d'un recours suivant l'article 227 L.A.U. Encore une fois cependant, cet argument ne pourra être considéré que si tous les critères liés à la demande de dérogation mineure ont effectivement été respectés et que cette option demeure ultimement réalisable ${ }^{74}$.

\section{D) LA PORTÉE DU POUVOIR DISCRÉTIONNAIRE EN MATIÈRE DE DÉROGATION MINEURE}

Outre les trois volets précités, il existe certaines conditions impératives devant guider l'examen d'une demande de dérogation mineure par le C.C.U. et le conseil municipal. D'abord, une telle demande devra respecter les objectifs du plan d'urbanisme ${ }^{75}$. Cet énoncé très libéral rend relativement «diaphane» ce critère compte tenu que seuls «les objectifs» du plan d'urbanisme sont visés par l'article 145.2 L.A.U. et non ses dispositions à proprement parler ${ }^{76}$.

Le conseil municipal qui adopte un règlement sur les dérogations mineures exerce un pouvoir purement discrétionnaire dans l'acceptation ou le refus d'une telle demande. Comme l'écrivait l'Honorable juge Tôth dans l'affaire Centre d'accueil Deauville Inc. c. Deauville (Corporation du village $d e)^{77}$ :

Certes la Loi prévoit certaines limites à la discrétion (usage et la densité d'occupation du sol, atteinte à la jouissance par les propriétaires des immeubles voisins, objectifs du plan d'urbanisme) et

74. Voir à cet effet les affaires Wentworth (Municipalité du canton de) c. Serre, J.E. 94-1662 (C.S.) et Saint-André-Avellin (Corporation municipale de la paroisse de), ibid,, où la Cour refusa de considérer cette alternative compte tenu qu' aucune demande de permis n'avait été soumise à la municipalité avant l'érection de la construction; voir l'affaire Beauport (Ville de) c. Fortier (3 décembre 1987), Québec 200-05-002284-870 (C.S.) [ci-après Beauport (Ville de)] où la Cour en arriva à la conclusion que dans un cas de bonne foi et en l'absence de préjudice, elle pouvait refuser d'ordonner la démolition de l'immeuble puisque la différence de hauteur était minime et qu'une dérogation mineure pouvait corriger la situation.

75. Art. 145.2 L.A.U.; Beauport (Ville de), ibid.; Auger c. Lévis (Ville de) (4 mai 1989), Québec 200-05-000899-977 (C.S.) [ci-après Auger].

76. $\quad$ Gauthier c. Hamilton (City of) (1987), 19 O.M.B.R. 457.

77. [1988] R.D.I. 347 (C.S.) [ci-après Centre d'accueil Deauville Inc.]. 
est subordonnée à la condition que le requérant en dérogation souffre un préjudice sérieux par l'application du règlement de zonage. À l'intérieur de ces limites, le pouvoir du conseil est discrétionnaire et ceci de par la nature même du pouvoir que le législateur a jugé bon de conférer aux corporations municipales. Cette discrétion, sauf le cas de violation de la loi, d'abus ou de fraude, échappe au contrôle des tribunaux.

Il apparaît néanmoins possible pour le conseil municipal de s'engager à l'avance à accorder une dérogation mineure si les conditions de délivrance du permis de construction sont respectées par le demandeur. C'est en effet la conclusion à laquelle en est arrivée la Cour supérieure dans l'affaire Girard c. Linteau ${ }^{78}$.

La dérogation mineure ne pourra être accordée «que si l'application du règlement a pour effet de causer un préjudice sérieux à la personne qui la demande ${ }^{79}$. Aussi, dans l'éventualité où cette personne peut réaliser son projet en se conformant à la réglementation applicable, il y a tout lieu de croire que cette demande devra être rejetée ${ }^{80}$.

La dérogation mineure ne pourra être accordée si elle porte atteinte à la jouissance, par les propriétaires des immeubles voisins, de leur droit de propriété $^{81}$. La Cour supérieure formulait l'opinion dans l'affaire Centre d'accueil Deauville Inc. ${ }^{82}$ qu'il ressortait du pouvoir du conseil municipal d'apprécier ce qui constitue une atteinte à la jouissance du droit de propriété des propriétaires voisins :

Si une telle dérogation nuit ou non aux voisins sont des questions de faits dont la constatation et leur appréciation dans leur contexte sont

78. J.E. 96-404 (C.S.).

79. L.A.U., supra note 1, art. 145.4 (2).

80. Jones c. Etobicoke (Borough of) (1983), 14 O.M.B.R. 128; Toronto (City of) c. Dahota (1986), 18 O.M.B.R. 62; Beauport (Ville de), supra note 74; Auger, supra note 75.

81. L.A.U., supra note 1, art. 145.4 (2).

82. Supra, note 77. Voir aussi Toronto (City of) c. Toronto Committee of Adjustments (1987), 33 M.P.L.R. 101 (Ont. M.B.); Y. Desjardins, «Dérogations mineures aux règlements d'urbanisme : pouvoir discrétionnaire» (1988-89), 91 R.du N. 373. 
du ressort du pouvoir discrétionnaire du conseil municipal et ne sont pas susceptibles d'être révisées par la Cour supérieure.

À cet égard, on peut prétendre que la tolérance pendant plusieurs années par les voisins d'une situation dérogatoire pourra être considérée par le conseil municipal dans l'étude du critère de «l'atteinte à la jouissance du droit de propriété» $^{83}$.

À la lumière de ce qui précède, il apparaît difficile de déterminer quels sont les voisins concernés par l'application de l'article 145.4 L.A.U. L'esprit de la loi tend cependant vers une participation communautaire accrue. En prenant en considération le caractère très libéral de l'expression «tout intéressée ${ }^{84}$ relative à la participation des citoyens lors de la présentation de la demande, il nous apparaît difficile de restreindre l'application de l'article 145.4 L.A.U. aux seuls propriétaires dont l'immeuble est immédiatement contigu à celui du demandeur.

\section{E) LA QUANTIFICATION DE LA DÉROGATION MINEURE}

L'expression «dérogation mineure» n'est nulle part définie à la Loi. Comme l'écrivait l'Honorable juge Orville Frenette dans l'affaire 151951 Canada Inc. c. Val-des-Monts (Municipalité de) ${ }^{85}$, citant $\mathrm{M}^{\mathrm{e}}$ François Robert :

L'expression «dérogation mineure» n'est pas définie dans la loi et sa portée exacte n'est pas précisée. La jurisprudence, à ce sujet, consacre la discrétion du conseil.

Après avoir analysé l'arrêt McNamara Corp. Ltd. c. Colekin Investments Ltd. émanant de l'Ontario, $\mathrm{M}^{\mathrm{e}}$ Robert ajoutait :

«Au Québec, il n'existe pas d'organisme contrôlant la discrétion du conseil comme en Ontario, sauf finalement les tribunaux. Ceux-ci sont cependant réticents à examiner

83. Auger, supra note 75.

84. L.A.U., supra note 1, art. 145.6 al. 2.

85. Supra note 72. 
ce qu'est une dérogation mineure au motif que ce pouvoir discrétionnaire appartient au conseil et ne peut pas être révisé par les tribunaux.»

Son interprétation est donc laissée à l'entière appréciation du conseil municipal, laquelle est toutefois encadrée par le contenu impératif du règlement sur les dérogations mineures ${ }^{86}$.

La jurisprudence à ce jour ne s'est guère prononcée sur la portée à donner à cette expression. Toutefois, la jurisprudence relative à l'article 227 L.A.U. regorge d'exemples où la Cour supérieure a exercé sa discrétion pour refuser de faire droit au recours sur la base de dérogations à la réglementation d'urbanisme dites «mineures». À cet égard, il est intéressant de noter que la Cour d'appel vint préciser dans l'affaire Boily c. Bélanger ${ }^{87}$ que le pouvoir donné au conseil municipal d'adopter un règlement sur les dérogations mineures n'a pas eu pour effet d'enlever la discrétion accordée à la Cour supérieure par l'article 227 L.A.U.

Ainsi, dans l'affaire Westmount (Ville de) c. Finkelstein ${ }^{88}$, la Cour refusa d'ordonner la démolition d'une construction compte tenu que l'élément dérogatoire constituait une contravention mineure de nature à faire l'objet d'une dérogation mineure. Cette orientation fut généralement maintenue par la Cour supérieure. Par exemple, dans l'affaire Beauport (Ville de) ${ }^{89}$, elle jugea mineure la dérogation quant à la hauteur excessive d'un toit alors que dans l'affaire Saint-Joseph (Corporation municipale de la paroisse de) c. Société québécoise d'assainissement des eaux ${ }^{90}$ elle considéra comme mineur le non-respect des normes d'éloignement d'un bassin d'aération des eaux usées par rapport aux habitations situées à proximité. Également, fut considéré mineur l'empiétement

86. L.A.U., supra note 1 , art. 145.3 .

87. [1989] R.J.Q. 572 (C.A.).

88. J.E. 84-779 (C.S.).

89. Supra note 74.

90. [1990] R.J.Q. 2004 (C.S.). 
de quelques pieds dans une marge de recul vu les conséquences dramatiques qui auraient découlé d'une correction de cette situation ${ }^{91}$.

À l'opposé, furent jugées des contraventions majeures à la réglementation d'urbanisme le fait d'opérer une entreprise de camionnage et d'entreposage de machinerie lourde dans une zone résidentielle ${ }^{92}$, la construction d'une route dans la zone inondable ${ }^{93}$, l'érection d'un garage dans la cour avant d'un terrain ${ }^{94}$, l'entreposage illégal de grues ${ }^{95}$, la construction de résidences en zones inondables par la transformation de bâtiments accessoires ${ }^{96}$, l'érection d'une clôture de deux mètres dans la cour avant alors que seules les clôtures d'une hauteur maximum d'un mètre étaient autorisées ${ }^{97}$ ainsi qu'un empiétement d'un mètre dans la bande de protection riveraine ${ }^{98}$.

Dans l'affaire précitée Parent c. Couture ${ }^{99}$, la Cour supérieure énonça quelques critères pouvant servir à déterminer le caractère mineur ou majeur de la dérogation à la réglementation. Selon cette opinion, "la nature, l'effet, l'importance de la dérogation, la bonne foi du contrevenant ainsi que le caractère préjudiciable de la démolition» sont autant de critères à appliquer dans la quantification de la dérogation. Il faut toutefois tenir compte que les articles 145.1 et suivants prévoient déjà certaines balises à l'exercice par le conseil municipal de son pouvoir discrétionnaire et que les critères précités ne peuvent s'appliquer qu'une fois l'avis du C.C.U. reçu, que l'analyse de la conformité aux objectifs du plan d'urbanisme a été complétée ainsi qu'une fois les intéressés entendus et que l'impact sur le droit de propriété des voisins s'est vu évalué ${ }^{100}$.

91. Kaczynski c. Bernier, J.E. 91-259 (C.S.); Irwin c. Baillargeon, J.E. 94-1879 (C.S.); Greenberg c. Gabriel, [1996] R.D.I. 26 (C.A.).

92. Laval (Ville de) c. Cardinal, J.E. 88-568 (C.S.).

93. Abitibi (Municipalité régionale de comté) c. Ibitiba Ltée, [1993] R.J.Q. 1061 (C.A.).

94. Stukely-Sud (Municipalité de) c. Labrèche, [1994] R.D.I. 90 (C.S.).

95. Sainte-Julie (Ville de) c. Entreprises Guay Inc., [1996] R.D.J. 246 (C.A.).

96. Parent c. Couture (22 août 1996), Québec 200-05-003722-969 (C.S.).

97. Port-Cartier (Ville de) c. Richard (10 janvier 1997), Mingan 650-05-000073-966 (C.S.).

98. $\quad$ Sainte-Catherine (Ville de) c. Gestion Seattle Inc., J.E. $94-1663$ (C.S.).

99. Supra note 96.

100. Voir à titre d'exemple en droit ontarien : McNamara Corp. Ltd. c. Colekin Investments Ltd., (1977) 2 M.P.L.R. 61 (C. Div. Ont) [ci-après McNamara Corp. Ltd]; Fisher c. Southworld (Township of) (1989), 22 O.M.B.R. 229. 
Enfin, il est intéressant de noter que la jurisprudence ontarienne est majoritairement d'avis que l'on ne peut, par le biais du processus de dérogation mineure, autoriser ou permettre ce qui est totalement interdit par la réglementation ou ce qui constitue une composante importante de l'exercice du droit de propriété ${ }^{101}$. Également, il ressort de cette jurisprudence que les multiples dérogations d'un projet unique devront faire l'objet d'une seule demande et d'un examen collectif de manière à circonscrire dans sa globalité la portée d'une telle demande ${ }^{102}$.

\section{F) LE CONTRÔLE JUDICIAIRE DES DÉCISIONS DU CONSEIL MUNICIPAL}

Comme nous le mentionnions précédemment, le conseil municipal jouit d'une grande discrétion dans l'appréciation d'une demande de dérogation mineure. Cette discrétion découle du texte habilitant de la L.A.U. Bien qu'une autorité réglementaire n'ait normalement pas «le droit de se réserver un pouvoir discrétionnaire d'accorder des dérogations à la règle générale dans des cas particuliers après avoir établi des normes objectives de comportements uniformes pour tous les administrés» ${ }^{103}$, elle pourra valablement agir ainsi du fait de l'habilitation précitée qui rend indubitable la qualité de son action. À cet égard, les auteurs Dussault et Borgeat écrivait ${ }^{104}$ :

Alors qu' on attend d'elle, sur certaines questions de détail non prévues par la loi, l'édiction de normes objectives de comportements uniformes pour tous les administrés, l'autorité réglementante s'accorde quelques fois, en décidant de rédiger les règlements en termes discrétionnaires, le pouvoir de rendre une décision particulière dans chaque cas. C'est le cas par exemple d'une régie qui, autorisée à réglementer les conditions d'obtention d'un permis, décide d'adopter un règlement lui accordant toute discrétion concernant l'émission de chacun des permis réclamés. L'illégalité d'un tel procédé, s'il n'est pas autorisé expressément par le Parlement, ne fait aucun doute; l'exercice d'un

101. McNamara Corp. Ltd., ibid.; Russel c. Munch \& Fun Amunseland (1987), 20 O.M.B.R. 162.

102. Toronto Committee of Adjustments, supra note 82.

103. 151951 Canada Inc., supra note 72.

104. R. Dussault et L. Borgeat, Traité du droit administratif, $2^{\mathrm{e}}$ éd., t. 1, Québec, Presses de l'Université Laval,1984 aux pp. 533-535. 
pouvoir réglementaire doit avoir pour objet l'adoption de normes générales et non l'établissement d'une discrétion administrative.

L'exercice du pouvoir discrétionnaire du conseil municipal demeure donc assujetti à l' application de certaines règles cardinales de droit administratif.

En matière municipale, les tribunaux ont tendance à respecter l'exercice par le conseil municipal d'un pouvoir discrétionnaire qui lui est dévolu par la loi. L'intervention des tribunaux se limite normalement à l'évaluation de motifs de légalité et non d'opportunité ${ }^{105}$. Toutefois, le pouvoir habilitant octroyant une certaine discrétion ne peut être indûment élargi. À cet effet, nous reprendrons l'énumération contenue à l'affaire 151951 Canada Inc. c. Val-des-Monts (Municipalité de) laquelle résume bien, à notre avis, la portée des règles cardinales dont nous faisions mentions plus haut :

1. Limites liées à la nature des pouvoirs des catégories : excès de pouvoir ou de juridiction, sous-délégation de pouvoir, réserve d'une discrétion administrative et imprécision;

2. Limites procédurales : l'omission de respecter les formalités essentielles et l'irrespect aux règles de la justice naturelle;

3. L'abus des pouvoirs, des fins abusives, la discrimination, la mauvaise foi et l'irrationalité. ${ }^{106}$

Le pouvoir d'accorder ou de refuser une dérogation mineure étant un pouvoir prévu à la Loi, il nous apparait difficile de trouver un argument à son encontre pouvant découler des «limites liées à la nature des pouvoirs des catégories». À l'égard des «limites procédurales», l'exercice se simplifie légèrement puisqu'il existe une procédure impérative devant être appliquée lors

105. Boisjoli c. Goebel, [1982] C.S. 1; Boucherville (Ville de) c. Jaybalt Corporation, [1965] C.S. 611; Association des contribuables de Frelighsburg c. Brome-Missisquoi (Municipalité régionale de comté), J.E. 92-1002 (C.S.); voir la décision dans l'affaire Boucherville (Ville de) c. Dessureault, J.E. 91-646 (C.S.) eu égard à la réserve des tribunaux en matière de dérogation mineure, telle décision ayant été confirmée à J.E. 94-891 (C.A.) sous un autre point.

106. Supra note 72 à la p. 839. 
d'une demande de dérogation mineure, que l'on pense seulement à l'obligation du conseil municipal de prendre avis auprès du C.C.U. ou d'entendre «tout intéressé». Selon la Cour d'appel dans l'affaire Gale c. Chicoutimi (Ville de) ${ }^{107}$, on ne peut prétendre à la violation des règles de justice naturelle lorsque la municipalité a suivi toutes les formalités entourant l'étude d'une demande de dérogation mineure et que le voisin, opposé à la demande, et bien que correctement avisé, a négligé de se présenter à la séance du conseil municipal pour faire valoir ses griefs.

"L'abus de pouvoirs, les fins abusives, la discrimination, la mauvaise foi et l'irrationalité» sont, pour leur part, des motifs donnant pleinement ouverture au pouvoir de surveillance et de révision de la Cour supérieure. Cette opinion est d'ailleurs soutenue par le professeur Giroux lorsqu'il mentionne que :

[...] même si la loi confie au conseil municipal l'appréciation des critères prévus aux dispositions de la L.A.U. sur les dérogations mineures, même si le conseil municipal jouit d'une grande discrétion dans leur appréciation et, même si les tribunaux ne peuvent substituer leur jugement à celui du conseil municipal sur une question d'opportunité, les dispositions actuelles de la L.A.U. démontrent que c'est l'intention du législateur de laisser le contrôle judiciaire s'exercer sur une décision d'un conseil municipal accordant une dérogation mineure. Ce contrôle peut s'exercer sur le caractère mineur de la dérogation mineure ainsi que sur les critères prévus à la loi ${ }^{108}$.

Bien que la jurisprudence en cette matière ne fasse pas légion, elle se fait néanmoins unanime. Comme nous le mentionnions précédemment, l'Honorable juge Tôth écrivait, dans une décision qui fait figure de principe :

Certes la loi prévoit certaines limites à la discrétion (usage et la densité d'occupation du sol, atteinte à la jouissance par les propriétaires des immeubles voisins, objectifs du plan d'urbanisme) et est subordonnée à la condition que le requérant en dérogation souffre un préjudice

107. J.E. 94-769 (C.A.).

108. Giroux, supra note 58 aux pp. 182-183. 
sérieux par l'application du règlement de zonage. À l'intérieur de ces limites, le pouvoir du conseil est discrétionnaire et ceci de par la nature même du pouvoir que le législateur a jugé bon de conférer aux corporations municipales. Cette discrétion, sauf le cas de violation de la loi, d'abus ou de fraude, échappe au contrôle des tribunaux ${ }^{109}$.

Au même effet, l'Honorable Juge Frenette écrivait dans la cause Gagné c. Lac Simon (Corporation municipale $d u)^{110}$ :

La jurisprudence énonce que les tribunaux ne doivent pas modifier les décisions des corporations municipales à moins qu'elles ne soient faites de mauvaise foi, discriminatoires, équivalant à fraude ou abus de pouvoir ou conduisant à une injustice flagrante. Les tribunaux ne contrôlent que la légalité de ces décisions et non pas leur opportunité.

Il faut donc retenir de ces décisions que la violation de la Loi, l'abus de droit, la mauvaise foi ou la fraude donnent ouverture au pouvoir de surveillance et de contrôle de la Cour supérieure. Conformément à l'opinion du professeur Giroux, nous voyons difficilement en quoi ce pouvoir pourrait être limité à l'exercice, à proprement parler, de l'activité discrétionnaire. À notre avis, la quantification de la dérogation par le conseil municipal nous apparaît être une composante de ce pouvoir pleinement assujettie au contrôle des tribunaux.

D'une manière plus générale, Louise Beaulieu, dans un texte publié en $1989^{111}$, résumait bien à notre avis les critères de validité de l'exercice discrétionnaire du conseil municipal. Avant d'analyser le critère de bonne foi, le caractère non arbitraire d'une décision ainsi que le caractère raisonnable d'une mesure adoptée par le conseil municipal, elle écrivait :

Le caractère discrétionnaire des actes d'une administration ou d'un fonctionnaire repose sur une marge de manoeuvre dont ceux-ci peuvent disposer en vertu d'une habilitation législative plus ou moins précise. Même lorsqu'un acte ou une décision est discrétionnaire, il

109. Centre d'accueil Deauville Inc., supra note 77 à la p. 349.

110. [1994] R.J.Q. 453 (C.S.) à la p. 458.

111. L. Beaulieu, «Les conditions de la validité d'un contrôle strict de l'utilisation du sol» (1989), 30 C. de D. 953. 
doit répondre à certaines exigences sous peine d'être contesté et éventuellement invalidé.

Cette opinion correspond au courant classique développé autour de la définition du mot «discrétion». Comme écrivait Lord Halsbury dans l'affaire Sharp c. Wakefield ${ }^{12}$ :

«[D]iscretion» means when it is said that some thing is to be done within the discretion of the authorities that that something is to be according to the rules of reason and justice, not according to private opinion : Rooke's Case (5 Rep. 100, A); according to law, and not humour. It is to be, not arbitrary, vague, and fanciful, but legal and regular. And it must be exercised within the limit, to which an honest man competent to the discharge of his office ought to confine himself : Wilson v. Rastall [4 T.R. at. p. 754].

En se fondant sur cette définition, force est de constater que si grande puisse être une discrétion conférée par le Parlement à une autorité administrative, celle-ci a quand même des limites.

Bien que les tribunaux se gardent d'intervenir dans l'exercice d'une discrétion administrative, le pouvoir discrétionnaire n'est pas illimité. Rappelons-nous à cet effet tout le contentieux entourant le principe de légalité. L'exercice d'un tel pouvoir s'inscrit habituellement dans le contexte de la loi habilitante.

Au Québec, l'affaire de principe en matière de contrôle de la discrétion administrative est l'arrêt Roncarelli c. Duplessis. Dans cette affaire, il s'agissait de déterminer s'il existait des limites au pouvoir discrétionnaire exercé par le responsable de la Commission de contrôle des permis d'alcool de retirer son permis à un détenteur. Le juge Rand de la Cour suprême s'exprimait ainsi :

In public regulation of this sort there is no such thing as absolute and untramelled «discretion», that is that action can be taken on any ground or for any reason that can be suggested to the mind of the 
administrator; no legislative Act can, without express language, be taken to contemplate an unlimited arbitraty power exercisable for any purpose, however capricious or irrelevant, regardless of the nature or purpose of the statute. Fraud and corruption in the Commission may not be mentioned in such statutes but they are always implied as exceptions. «Discretion» necessarily implies good faith in discharging public duty; there is always a perspective within which a statute is intended to operate; and any clear departure from its lines or objects is just as objectionable as fraud or corruption. ${ }^{113}$

Il est intéressant de noter que dans cette même décision, la Cour suprême du Canada discutait de la question de la poursuite d'une finalité autre que celle de la Loi, en ces termes ${ }^{114}$ :

(...) the grounds for refusing or cancelling a permit should unquestionnably be such and such only as are incompatible with the purposes envisaged by the statute : the duty of a Commission is to serve those purposes and those only. A decision to deny or cancel such a privilege lies within the «discretion» of the Commission; but that means that decision is to be based upon a weighting of consideration pertinent to the object of tha dministration (...).

Enfin, quant à la bonne foi, la Cour s'exprimait ainsi ${ }^{115}$ :

Good faith in this context, applicable to both the respondent and the general manager, means carrying out the statute according to its intent and for its purpose; it means good faith in acting with a rational appreciation of that intent and purpose, and not with an improper intent or for an alien purpose; it means good faith in acting with a rational appreciation of that intent and purpose and not with an improper intent or for an alien purpose; (...).

113. [1959] R.C.S. 121

114. Voir aussi : Lajeunesse c. Montréal (Cité de), [1963] C.S. 364.

115. Voir aussi : Hertel Holdings Co. c. Calgary (Ville de), [1984] 1 R.C.S. 337 confirmant (1982), 131 D.L.R. $3^{\mathrm{e}}$ ) 723 [ci-après Hertel Holdings Co.]. 


\section{SECTION III - IES PLANS D'AMÉNAGEMENT D'ENSEMBLE}

\section{A) LE ZONAGE DIFFÉRÉ («HOLDING BY-LAW»OU «SITE PLAN BY-LAW»)}

La réglementation détaillée d'une portion d'un territoire municipal non développé s'est toujours avérée une entreprise artificielle et parfois irréaliste. En fait, l'obligation dévolue au conseil municipal d'adopter des normes réglementaires objectives ne comportant l'exercice d'aucune discrétion s'apparentait plutôt à un véritable exercice de divination ${ }^{116}$. Dans l'objectif de rendre l'exercice de son pouvoir réglementaire plus adapté à ses besoins réels, l'administration municipale développa peu à peu un «remède» à l'obligation de «tout prévoir tout de suite»: le zonage différé. Comme son nom le dit, le zonage différé a comme particularité de retarder l'adoption de normes réglementaires détaillées pour une portion donnée du territoire municipal.

L'obligation de réglementer tout le territoire municipal demeure néanmoins applicable puisque la technique du zonage différé ne permet pas d'interdire toute utilisation du sol. Une telle prohibition pourrait constituer, dans les faits, une expropriation déguisée ${ }^{117}$. C'est pourquoi on prévoira normalement pour un secteur «vierge» la possibilité d'exercer des usages qui ne sont pas de nature à compromettre l'objectif de développement global préconisé par la municipalité. Par exemple, pour la période qui précède l'élaboration d'un plan de développement, la municipalité pourra prévoir dans ses règlements d'urbanisme que seuls des usages extensifs tels l'habitation à très faible densité ou l'agriculture sont autorisés. La nature peu attrayante et peu rentable de ces usages, qui ne paralysent toutefois pas complètement le développement de la portion de territoire placé "sous réserve», limite toutefois considérablement

116. P. Kenniff, «Le contrôle public de l'utilisation du sol et des ressources en droit québécois II» (1976), 17 C. de D. 85.

117. Voir L. Beaulieu, «Les conditions de la validité d'un contrôle strict de l'utilisation du sol» (1989), 30 C. de D. 953. 
toute intervention de nature à compromettre un développement complet et rentable ${ }^{118}$.

Comme nous le mentionnions précédemment, le zonage différé n'est pas né, au Canada, de l'initiative du législateur, mais plutôt de celle des municipalités ${ }^{119}$. Bien que cette technique de planification du développement territorial municipal n'ait pas toujours reçu l'aval des tribunaux ${ }^{120}$, elle fut cependant consacrée par la Cour suprême du Canada dans l'affaire Sanbay Developments Ltd. c. London (Corporation de la Ville de) ${ }^{121}$. Reconnaissant à l'autorité municipale le pouvoir et le droit «de geler» le développement de certaines portions de son territoire, la Cour écrivait :

Il a été reconnu de part et d'autre par les avocats que la municipalité est libre de geler l'aménagement et même d'exiger des règlements particuliers quant à des aménagements sur des parcelles de terrains différentes.

$[\ldots]$

La politique poursuivie par la Ville intimée est suffisamment claire, et on ne saurait aucunement mettre en question la liberté qu'elle a de l'adopter. Le fait qu'elle aurait pu faire plus nettement ce qu'elle a fait

118. F. Marchand, «Le zonage différé» (1985), 45 R. du B. 426; J. L’Heureux, Droit municipal québécois, t. 2, Montréal, Wilson \& Lafleur et SOREJ, 1984 au par. 1294; voir aussi Kenniff, «Approche réglementaire de l'aménagement urbain», supra note 58.

119. P.A. Gall, «Land Use Regulation - Holding Zones - Discretionary Development Control» (1979), 13 U.B.C.L. 409 : «Historically, zoning has been the primary mechanism for regulating land use and development in North America. It entails the division of areas into zones and the enactment of different restrictions for each zone. While the standard zoning by-law has proven effective in protecting stable neighbourhoods, it has been criticized as lacking the necessary flexibility to deal with areas undergoing change. As a result newer, more flexible methods of land use control have been increasingly utilized. One such device is the "holding zone", a land use category in which the restrictions imposed are considered to be only temporary. This technique is used for three basic purposes : to prevent premature development, to provide time for the formulation of planning policies, and to allow for control over individual development proposals.».

120. Regina c. King (Township of) ex p. O'Connor (1970), 15 D.L.R. (3 $\left.{ }^{\mathrm{e}}\right) 543$ (H.C. Ont.); Re Anzil Construction and West Gwillimbury (Township of) (1971), 19 D.L.R. (3 $\left.3^{\mathrm{e}}\right) 37$ (H.C. Ont.); Karamanolis c. Port Coquitlam (City of) (1979), 8 B.C.L.R. 282 (C.A.C.-B.).

121. [1975] 1 R.C.S. 485. 
ne signifie pas que la substance de sa politique de gel est en doute sous le régime des modifications apportées à son règlement général de zonage 306-480.

C'est également en 1975 que la Cour suprême du Canada rendit son jugement dans la célèbre affaire Soo Mill \& Lumber Co. c. Sault Sainte-Marie (Corporation de la Ville de) ${ }^{122}$ à l'occasion de laquelle elle écrivait :

Le fait de geler l'aménagement, conformément aux préceptes du Plan officiel mis à exécution par le règlement de zonage, n'équivaut pas à une violation du par. (1) de l'art. 35 lorsque des utilisations agricoles et connexes (et des utilisations préexistantes) sont permises. L'appelante ne peut pas non plus se plaindre de discrimination simplement parce que la conséquence du gel est la stérilité de son terrain du point de vue aménagement, lorsque cela intervient dans le contexte d'un Plan d'ensemble officiel et d'un règlement général de zonage adopté pour le mettre en oeuvre. Il n'y a pas eu d'allégation de mauvaise foi de la part de l'intimée lorsqu'elle a mis le terrain de l'appelante dans la «catégorie réservée». La municipalité avait discrétion pour ce faire en vertu du plan de zonage.

Bien plus que la simple reconnaissance du pouvoir municipal de réglementer par zonage différé, ces décisions ont ni plus ni moins orienté la perception et défini la perspective de l'expression «droit de propriété». Faisant suite à la décision rendue par cette même Cour en 1974 dans l'affaire Association des propriétaires des Jardins Taché Inc. c. Entreprises Dasken Inc. ${ }^{123}$, ces décisions allaient, en effet, être à l'origine d'un courant d'interprétation communautariste ayant pour essence de «situer» le sacro-saint droit de propriété, concept libéral par excellence, à l'intérieur de l'intérêt de la communauté ${ }^{124}$.

122. [1975] 2 R.C.S. 78.

123. [1974] R.C.S. 2.

124. Mascouche (Ville de) c. Thiffault, J.E. 96-1097 (C.A.); Mirabel (Ville de) c. Filiatreault (29 août 1984), Terrebonne (Saint-Jérôme) 700-05-000794-846 (C.S.); Saint-Romuald d'Etchemin (Corporation municipale de la cité de) c. Bisson, [1985] C.S. 84; Re Bruce and City of Toronto (1971), 3 O.R. 62; Drummondville (Cité de) c. Duchesne (8 novembre 1976), Québec 200-09-000007-741 (C.A.), j. Owen, Lajoie et Béanger; Heritage Trust of Nova Scotia c. Nova Scotia (Utility \& Review Board) (1994), 20 M.P.L.R. (2e) 84 (C.A.N.- 
Loi sur l'aménagement et l'urbanisme

La légalité du zonage différé fut par ailleurs confirmée de nouveau en 1984 par la Cour suprême du Canada dans l'affaire Hartel Holdings and City of Calgary $^{125}$.

Le zonage différé fut reconnu au Québec en 1985 dans l'affaire SaintRomuald-d'Etchemin (Corporation municipale de la Cité de) c. Bisson ${ }^{126} \mathrm{dont}$ le passage suivant, fort pertinent, mérite retranscription à titre de conclusion à notre commentaire :

[L]e «zonage différé» peut se caractériser comme étant un règlement temporaire dont le seul but est de limiter le développement dans certaines zones où l'état du développement ne permet pas de définir avec exactitude la nature, laissant à la municipalité le pouvoir de disposer de chaque demande d'amendement au profit d'un aménagement ultérieur répondant davantage à la réalité urbaine et à l'intérêt public.

$[\ldots]$

La Cour suprême a reconnu à deux reprises la validité juridique d'une pareille réglementation adoptée par des villes ontariennes dans les arrêts Sanbay Developpements Ltd. c. Corp. de la Ville de London et Soo Mill \& Lumber Co. c. Corp. de la Ville de Sault Sainte-Marie.

É.); Galway \& Cavendish (United Township) c. Windover (1996), 30 M.P.L.R. (2e 109 (Div. Gén. Ont.); P.A. Côté et J.-M. Delacoste, «Le système judiciaire et l'espace urbain» (1981-82), 16 R.J.T. 237; P.A. Gall, «Land Use Regulation - Holding Zones - Discretionary Development Control» (1979), 13 U.B.C.L. 409.

125. Supra note 115 .

126. [1985] C.S. 84 aux pp. 88-89, 91; voir également la décision dans Bourgie c. Association des Vinciaturesi de Montréal Inc., [1982] C.S. 994 suivant laquelle on ne peut isoler un des éléments qui doit faire partie d'un plan d'aménagement d'ensemble et prétendre pouvoir déposer un tel plan à l'égard de ce seul élément; Bilodeau c. Sainte-Foy (Ville de), J.E. 85852 (C.S.) et Tremblay c. Chicoutimi (Ville de), J.E. 84-894 (C.S.). Bien que ces trois décisions traitent du zonage différé et furent rendues avant l'affaire Saint-Romualdd'Etchemin c. Bisson, il faut noter que la validité de la technique du zonage différé n'y fut pas mise en doute. 
Dans l'affaire Sanbay, la Cour suprême a reconnu la légalité d'un règlement de zonage appelé «holding regulation» qui prohibait toute transformation ou érection.

«en vue de contenir trois logements ou plus tant que le présent règlement n'aura pas été modifié par l'adjonction d'annexes et d'appendices appropriés définissant le secteur et illustrant les secteurs permis de construction, de stationnement, de surface libre utilisable, ainsi que l'architecture permise, assorties de règles régissant la dimension, l'aire de plancher, les traits distinctifs et l'utilisation du bâtiment ou de la construction.»

Par ailleurs, dans l'affaire Soo Mill, la Cour suprême a reconnu à nouveau la légalité d'une disposition semblable qui interdisait pour les terrains compris dans une zone et dont le symbole zonal était suivi du suffixe $\ll \mathrm{H} »$ (pour holding category),

«d'y ériger ou utiliser des bâtiments, pour quelque fin que ce soit, si ce n'est pour les fins suivantes :

- 1 Toute utilisation en cours dudit terrain ou d'un bâtiment y érigé, le jour de l'adoption du présent règlement,

- 2 agriculture;

- 3 grande culture;

- 4 culture maraîchère et florale, pour le marché;

- 5 pâturage des chevaux, du bétail ou des moutons;

- 6 étal temporaire pour la vente de produits de ferme produits sur place;

- 7 occupation domestique;

- 8 utilisation accessoire...» 
[L]a Cour d'appel d'Alberta a reconnu la validité juridique d'un règlement de zonage différé et l'applicabilité des décisions de la Cour suprême et ce, en l'absence de toute disposition semblable au paragraphe 22 de l'article 35 du Ontario Planning Act dans la loi albertaine.

\section{B) L'AVÈNEMENT DES PLANS D'AMÉNAGEMENT D'ENSEMBLE (P.A.E.)}

Le législateur intervint en 1987 pour modifier la L.A.U. et y introduire une nouvelle section intitulée «Les plans d'aménagement d'ensemble» ${ }^{127}$. Le but visé par l'inclusion de cette section était de «mieux encadrer le pouvoir $d u$ conseil d'une municipalité de procéder au zonage d'une aire délimitée de son territoire par étape» ${ }^{128}$. Ainsi furent introduits dans la L.A.U. les articles 145.9 à 145.14 , lesquels donnèrent clairement aux municipalités le pouvoir d'exiger un P.A.E. dans le cadre d'un zonage différéé ${ }^{129}$.

Suivant l'article 145.9 L.A.U., le conseil municipal possède le pouvoir d'adopter un règlement qui lui permet d'exiger la production d'un P.A.E. lorsqu'une demande de modification à la réglementation d'urbanisme est présentée pour une zone donnée visée par ce règlement et non lors d'une demande de permis de construction. Le contexte de l'article implique

127. Loi modifiant la Loi sur l'aménagement et l'urbanisme, L.Q. 1987, c. 53.

128. Voir les notes explicatives du Projet de loi 43.

129. Voir à ce sujet : F. Marchand, «Chroniques. Le zonage différé» (1985), 45 R. du B. 426-428; M. Delorme, «Les plans d'aménagement d'ensemble et les plans d'implantation et d'intégration architecturale» dans Formation permanente du Barreau du Québec, Récents développements en droit municipal, Cowansville (Qc), Yvon Blais, 1992 aux pp. 49-66; Ministère des Affaires municipales, Direction générale de l'urbanisme et de l'aménagement du territoire, Les plans d'aménagement d'ensemble, 1987; Y. Duplessis et J. Hétu, «La loi sur l'aménagement et l'urbanisme» dans Chambre des notaires du Québec, Répertoire de droit, Montréal, 1991 aux pp. 189-192; voir S. M. Makuch, «Zoning : Avenues of Reform» (1973-74), 1 Dal. L.J. 294 à l'égard de l'actuelle mixité de notre régime juridique. Autrefois caractérisé par la stabilité de son zonage classique, il a depuis été introduit en matière de zonage des mécanismes destinés à assouplir et à améliorer le zonage (le P.A.E. par exemple). Ces normes apparaissent fortement inspirées du Development Control de l'Angleterre qui est à l'opposé du zonage classique empreint d'une grande objectivité. 
nécessairement, à notre avis, qu'il est nécessaire, dans le cas des P.A.E., de référer aux zones prévues au plan de zonage de la municipalité adopté en vertu de l'article 113 alinéa 2 paragraphe 1. En effet, bien que le texte de la Loi soit plutôt obscur à cet égard, par opposition à la clarté de l'article 145.3 L.A.U., la pratique ainsi que la logique du mécanisme nous enseignent qu'il ne saurait être autrement. Soulignons que le plan d'urbanisme de la municipalité pourra également prévoir des aires d'aménagement pouvant faire l'objet d'un P.A.E. La délimitation de telles aires est cependant facultative selon l'article 84 (7) L.A.U., sauf si le conseil de la municipalité régionale de comté oblige une municipalité, par résolution, à inclure dans son plan d'urbanisme de telles aires $^{130}$.

L'adoption de ce règlement est discrétionnaire, bien qu'assujettie à la procédure de consultation publique prévue aux articles 123 et suivants L.A.U. et exige la constitution préalable d'un C.C.U. Par ailleurs, rien n'empêche la municipalité d'intégrer à même son règlement de zonage les dispositions relatives au P.A.E., ce qui s'avère être souvent le cas en pratique.

C'est l'article 145.10 L.A.U. qui détermine le contenu obligatoire du règlement sur les P.A.E., lequel se divise en cinq rubriques :

$1^{\circ}$ indiquer la zone à l'égard de laquelle une modification des règlements d'urbanisme est assujettie à la production d'un plan d'aménagement d'ensemble;

$2^{\circ}$ spécifier, pour cette zone, les usages et les densités d'occupation du sol applicables à un plan d'aménagement d'ensemble;

$3^{\circ}$ établir la procédure relative à une demande de modification des règlements d'urbanisme lorsque la présentation d'un plan d'aménagement d'ensemble est requise;

$4^{\circ}$ prescrire les éléments qu'un plan d'aménagement d'ensemble doit représenter et les documents qui doivent l'accompagner;

$5^{\circ}$ déterminer les critères suivant lesquels est faite l'évaluation d'un plan d'aménagement d'ensemble. ${ }^{131}$

130. L.A.U., supra note 1 , art. 86.

131. Ibid., art. 145.10. 
David Belgue définissait ainsi le contenu de ces rubriques dans une publication du ministère des Affaires municipales ${ }^{132}$ :

\section{Les usages et les densités applicables}

Le plan et les règlements d'urbanisme en vigueur spécifient les usages et les densités autorisés avant la production d'un P.A.E. Mais, afin d'informer des promoteurs de la vocation ultérieure de la zone, le règlement sur les P.A.E. doit spécifier les usages et densités éventuels suite à la modification des règlements d'urbanisme. Par exemple, dans une zone périphérique où la réglementation actuelle permet l'agriculture ainsi que l'habitation à très faible densité et sans services, le règlement sur les P.A.E. pourrait indiquer que la municipalité entrevoie le développement d'un quartier résidentiel complet avec l'habitation à faible, moyenne et forte densité, le commerce de quartier, les espaces verts, les équipements récréatifs, etc.

Toutefois, ces usages et densités futurs ne seront pas localisés sur plan ni quantifiés sous forme de superficies; cette planification étant différée jusqu'au moment de la production du P.A.E.

\section{La procédure}

En plus des étapes prévues par la loi, la municipalité peut identifier le fonctionnaire à qui le P.A.E. doit être transmis, la possibilité de soumettre un avant-projet, les mécanismes supplémentaires de consultation, etc.

\section{Les éléments du plan et les documents d'accompagnement}

Il s'agit d'abord des éléments devant paraître sur le plan, tels que les affectations du sol, les densités, le réseau routier, l'emplacement approximatif des bâtiments, l'aménagement paysager, etc. L'échelle du plan peut également être spécifiée. En ce qui concerne les documents accompagnant le plan, ceux-ci peuvent inclure des plans et élévations des bâtiments, le bordereau des superficies, la description des plantations à réaliser, etc.

132. Ministère des Affaires municipales, Direction générale de l'urbanisme et de l'aménagement du territoire, Les plans d'aménagement d'ensemble, 1987, Québec. 
Chaque municipalité peut définir le niveau de détail qu'elle souhaite dans les P.A.E., compte tenu des zones concernées et de ses préoccupations. Évidemment, il doit exister une relation étroite entre les éléments du plan demandés et les critères d'évaluation puisque le plan doit contenir l'ensemble des éléments nécessaires à cette évaluation.

\section{Les critères d'évaluation}

L'énumération des critères dans le règlement a deux buts :

- Énoncer clairement pour les éventuels promoteurs susceptibles de préparer un P.A.E. pour une zone donnée, les attentes de la municipalité concernant la nature et les caractéristiques souhaitées du développement (ces critères s'ajoutent à l'identification des usages et densités en vertu du $2^{\circ}$ alinéa de l'article 145.10).

- $\quad$ Fournir à l'avance les critères qui serviront à l'évaluation par les services techniques, le comité consultatif d'urbanisme et le conseil de chaque P.A.E.

Ces critères doivent comprendre tous les aspects que la municipalité considère comme importants (ex. : la conservation d'un boisé existant, la séparation des usages incompatibles, la protection des droits de vue et d'ensoleillement, les matériaux de revêtement à privilégier).

Ces critères peuvent être exprimés sous forme d'objectifs qualitatifs à rencontrer (ex. : les espaces verts devraient être intégrés à un réseau de voies piétonnières) ou d'objectifs quantitatifs (ex. : au moins $40 \%$ de la superficie boisée devrait être conservée).

De plus, certaines normes utilisées dans d'autres zones ou dans l'ensemble du territoire municipal peuvent s'appliquer dans les zones de P.A.E. (ex. : le nombre d'espaces de stationnement requis pour chaque usage $)^{133}$.

133. Voir à cet égard nos commentaires relativement au contrôle judiciaire des décisions du conseil municipal dans la section traitant des dérogations mineures, ci-dessus section II- F). 
De par sa nature donc, le règlement sur les P.A.E. reprend l'essence du zonage différé tel que développé par les tribunaux au fil des ans. D'ailleurs, la Cour suprême du Canada s'exprimait ainsi à l'égard des P.A.E. dans l'affaire Lalande c. Québec (P.G. $)^{134}$ :

[Le règlement sur les plans d'aménagement d'ensemble] permet de contourner les rigueurs des autres règlements de zonage, de construction, de lotissement et de permis et, avec un peu d'imagination et de bonne volonté, de convenir d'un projet de construction qui satisfasse à la foi aux désirs matériels légitimes des requérants et aux contraintes gouvernementales. [...] [U]n plan d'aménagement d'ensemble n'est pas une contrainte ni un carcan mais un appel à la concertation, à la discussion et au compromis.

Par ailleurs, compte tenu que la question fut posée en Commission parlementaire, il y a lieu de préciser que «la procédure relative à une demande de modification des règlements d'urbanisme» prévue au paragraphe 3 de l'article 145.10 L.A.U. ne concerne que la présentation du P.A.E. et non la procédure de consultation sur la modification des règlements d'urbanisme, laquelle est déjà prévue aux articles 123 et suivants L.A.U.

\section{C) LES CONDITIONS D'APPROBATION D'UN PLAN D'AMÉNAGEMENT D'ENSEMBLE}

Le conseil municipal d'une municipalité peut exiger, comme condition d'approbation d'un P.A.E., que les propriétaires des immeubles situés dans la zone visée par le plan :

$1^{\circ}$ prennent à leur charge le coût de certains éléments du plan, notamment des infrastructures et des équipements;

$2^{\circ}$ réalisent le plan dans le délai qu'il impartit;

$3^{\circ}$ fournissent les garanties financières qu'il détermine ${ }^{135}$.

134. [1991] R.J.Q. 1629 (C.S.).

135. L.A.U., supra note 1, art. 145.13 . 
La construction des infrastructures représente une dépense importante. Par l'article 145.13, la municipalité possède donc le pouvoir d'assujettir l'approbation du P.A.E. à la participation financière du promoteur ou à la prise en charge complète du coût des infrastructures. La réalisation du P.A.E. dans un délai donné vise, pour sa part, deux objectifs particuliers. D'une part, la détermination d'un délai de réalisation évite une spéculation effrénée découlant d'un changement de zonage bénéfique sur la valeur des terrains. Il faut en effet prendre pour acquis que la municipalité verra à agir très rapidement pour modifier sa réglementation de manière à y inclure le P.A.E. et à le rendre opérationnel dans les meilleurs délais. D'autre part, un tel délai permet à la municipalité d'anticiper le versement de taxes municipales dans un avenir déterminé.

Quant à la condition liée aux garanties financières, elle vient compléter le pouvoir dont sont déjà pourvues les municipalités par l'article 470 L.C.V. Cette condition vise à assurer aux municipalités une certaine garantie d'exécution ainsi que la présence de moyens concrets pour poursuivre le développement du P.A.E. ou se faire indemniser en cas de non-respect des conditions d'approbation.

Puisque c'est par résolution que le conseil municipal approuvera ou refusera le P.A.E. selon l'article 145.12 L.A.U., il y a tout lieu de croire que les conditions d'approbation précitées y seront énoncées ou seront contenues dans une entente particulière à laquelle réfèrera en détail la résolution. Cette résolution devra être transmise à la personne qui a présenté le P.A.E.

Finalement, la municipalité pourra adopter un projet de règlement visant l'inclusion à la réglementation d'urbanisme du P.A.E. Celui-ci étant dans la plupart des cas d'une grande précision et s'avérant, de plus, complètement adapté au territoire qu'il vise, il constitue sans contredit la meilleure réglementation applicable. Aussi, grâce à cette modification à la réglementation d'urbanisme, le P.A.E. sera opposable à tous les propriétaires de la zone et s'appliquera lors de l'émission éventuelle d'un permis de construction ou de lotissement. Le P.A.E. faisant désormais partie de la réglementation d'urbanisme, il pourra, dans la mesure de la loi, faire l'objet de dérogations mineures si certaines portions des travaux s'avèrent difficiles à exécuter. 
Dans l'éventualité où un règlement sur les P.A.E. est adopté, il ne pourra être interprété comme constituant un contrat avec la municipalité. En effet, celle-ci ne peut renoncer à son droit éventuel d'abroger un règlement et lier ainsi les conseils futurs ${ }^{136}$. De plus, il faut noter que la Commission municipale n'a pas compétence pour se prononcer sur la validité d'un règlement découlant des articles 145.9 et suivants ${ }^{137}$.

\section{SECTION IV - L ES PLA NS D' I M P L A N T A T I O N E T D'INTÉGRATION ARCHITECTURALE (P.I.I.A.)}

\section{A) ORIGINE ET NATURE}

C'est dans la foulée de l'inclusion à la L.A.U. de concepts discrétionnaires visant à assouplir les normes de zonage que le législateur adoptait, en 1989, la Loi modifiant la Loi sur l'aménagement et l'urbanisme et d'autres dispositions législatives ${ }^{138}$.

Un des effets de cette loi fut d'ajouter à la L.A.U. une section complète, soit les articles 145.15 à 145.20 , portant sur les P.I.I.A. ${ }^{139}$. D'inspiration américaine, le concept des P.I.I.A. fut développé autour de concepts retenus par les tribunaux américains pour limiter et encadrer la discrétion du conseil municipal $^{140}$.

136. Desrosiers c. Longueuil (Ville de), J.E. $81-593$ (C.S.).

137. Cormier c. L'Assomption (Ville de) (1994), 11 C.M.Q. 881.

138. L.Q. 1989, c. 46.

139. J.-P. St-Amour, «Les plans d'implantation et d'intégration architecturale» (1994), 1 B.D.M. 74-76, 86-89; Ministère des Affaires municipales, Direction générale de l'urbanisme et de l'aménagement du territoire, Les plans d'implantation et d'intégration architecturale, 1989, Québec; Les plans d'implantation et d'intégration architecturale - Guide explicatif, 1994, Québec.

140. $\quad \mathrm{M}^{\mathrm{e}}$ Michel Delorme, rapportant les propos de David Belgue, résume en cinq points ces critères : " $1^{\circ} L$ 'autorisation d'exercer une telle discrétion doit être expressément autorisée par la loi.

$2^{\circ}$ Cette discrétion doit se faire en fonction de critères précis d'évaluation;

$3^{\circ}$ Elle doit également s'exercer dans le cadre d'une procédure équitable incluant à titre d'exemple, l'obligation de rendre des décisions motivées, la possibilité d'appel, la possibilité de se faire entendre, etc..

$4^{\circ}$ Les règles d'évaluation doivent être claires : documents explicatifs, délais raisonnables de prise de décisions, etc. 
En ce qui a trait aux autres provinces canadiennes, la plupart s'étaient depuis quelques années déjà pourvues de dispositions semblables à celles maintenant en vigueur au Québec ${ }^{141}$.

\section{B) LE CHAMP D'APPLICATION}

Comme c'est le cas pour les P.A.E. et les dérogations mineures, l'adoption d'un règlement sur les P.I.I.A. est d'abord assujettie à la constitution d'un C.C.U. L'adoption de ce règlement n'a pas pour objectif de contrôler l'opportunité du développement ou des travaux envisagés, mais plutôt leur apparence et leur intégration architecturale dans un milieu bâti ou non. Il s'agit donc d'encadrer par l'exercice d'un pouvoir discrétionnaire l'exécution de travaux et non de soumettre l'opportunité de les réaliser au bon vouloir du conseil municipal. Il va de soi que l'adoption d'un tel règlement est purement discrétionnaire bien qu'elle soit soumise à la procédure de consultation publique prévue aux articles 123 et suivants L.A.U. Cependant, par application des articles 48, 53.10, 58, 59, 59.1, 59.5 et 59.6 L.A.U., une municipalité régionale de comté ayant modifié ou révisé son schéma d'aménagement peut exiger la concordance avec ce dernier du règlement sur les P.I.I.A. des municipalités locales. Elle peut également, à l'inverse, soustraire ce règlement de l'examen de conformité au schéma par l'application de l'article 237.2 L.A.U.

Elle ne peut, toutefois, par le biais de son schéma, exiger qu'une municipalité locale ait recours à la technique des P.I.I.A. pour un secteur donné

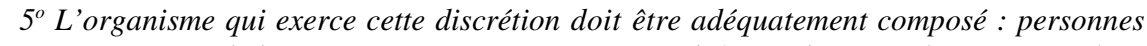
représentatives de la communauté, experts, etc.»; (Voir à ce sujet M. Delorme, «Les plans d'aménagement d'ensemble et les plans d'implantation et d'intégration architecturale» dans Formation permanente du Barreau du Québec, Développements récents en droit municipal, Cowansville (Qc), Yvon Blais, 1992, 49 aux pp. 49-66; Ministère des Affaires municipales, Direction générale de l'urbanisme et de l'aménagement du territoire, Les plans d'implantation et d'intégration architecturale,1989, Québec.

141. Voir à cet effet M. Osbaldeston, «Aesthetics and the legal Regulation of Architecture in Ontario» (1993), 15 M.P.L.R. (2 $\left.2^{\mathrm{e}}\right) 74$ à la p. 76 : «Design control (beyond bulk and spacing) is not a traditional area of municipal zoning regulation, although it has unquestionably developed in some Canadian jurisdictions. But in those jurisdictions that do allow design control, the empowering language is explicit, in one instance allowing regulation for «the architectural design or external appearance of structures» (Nova Scotia), and in two instances for «design, character and appearance» (New Brunswick and Alberta)». 
de la M.R.C. De la même manière, il n'existe aucune obligation pour la municipalité d'identifier au plan d'urbanisme les parties de son territoire qui sont assujetties à la production de P.I.I.A. Le dépôt d'un avis de motion en vue d'adopter ou de modifier un règlement sur les P.I.I.A. comporte le même «effet de gel» sur l'émission des permis et certificats que l'avis de motion donné en vue d'adopter ou de modifier un règlement de zonage ${ }^{142}$ ou de lotissement ${ }^{143}$.

Une politique d'intégration des constructions est donc le fer de lance d'une telle réglementation. Pour ce faire, le conseil municipal doit établir et déterminer avec la plus grande clarté les objectifs qu'il vise à atteindre par l'adoption d'un tel règlement qui soumet l'émission d'un permis ou certificat à des critères supplémentaires par ailleurs discrétionnaires.

Il faut noter que tous les permis et certificats prévus aux articles 119 à 122 L.A.U. sont visés par l'article 145.15 L.A.U. De plus, compte tenu que le vocabulaire utilisé à cet article est excessivement libéral, il y a lieu de croire que tous les types de travaux préalablement assujettis à l'obtention d'un permis ou d'un certificat, que l'on songe seulement à la démolition, à la construction ou à l'agrandissement d'une construction, à la transformation par remblai d'un terrain ou aux opérations cadastrales, peuvent faire l'objet d'un P.I.I.A.

\section{C) LE CONTENU DU RÈGLEMENT SUR LES PLANS D'IMPLANTATION ET D'INTÉGRATIONARCHITECTURALE}

Selon l'article 145.16 L.A.U., le règlement sur les P.I.I.A. doit prévoir le détail des rubriques suivantes :

$1^{\mathrm{o}}$ indiquer toute zone ou catégorie de constructions, de terrains ou de travaux visée;

$2^{\circ}$ déterminer les objectifs applicables à l'implantation et à l'architecture des constructions ou à l'aménagement des terrains, ainsi que les critères permettant d'évaluer si ces objectifs sont atteints;

$3^{\circ}$ prescrire le contenu minimal des plans et exiger, notamment, qu'ils contiennent l'un ou plusieurs des éléments suivants :

142. L.A.U., supra note 1 , art. 114.

143. Ibid., art. 117. 
a) la localisation des constructions existantes et projetées;

b) l'état du terrain et l'aménagement qui en est projeté;

c) l'architecture des constructions qui doivent faire

l'objet de travaux de construction, de transformation, d'agrandissement ou d'addition;

d) la relation de ces constructions avec les constructions adjacentes;

$4^{\mathrm{o}}$ prescrire les documents qui doivent accompagner les plans;

$5^{\circ}$ prescrire la procédure relative à la demande de permis de construction ou de lotissement ou à la demande de certificat d'autorisation ou d'occupation lorsque la délivrance de ce permis ou certificat est assujettie à l'approbation des plans.

En pratique, le mot «zone» prévu au paragraphe 1 n'est pas interprété comme faisant référence aux zones prévues au plan de zonage de la municipalité. Suivant cette interprétation, la division du territoire de la municipalité peut donc se distinguer, aux fins du règlement sur les P.I.I.A., du découpage prévu au plan de zonage. Cette interprétation nous semble par ailleurs contraire à la règle de droit voulant qu'un terme utilisé à plusieurs reprises dans une loi soit toujours sujet à la même interprétation, à moins de spécification au contraire à cet effet. D'aucuns pourraient prétendre que contrairement à l'article 145.3 L.A.U. relatif aux dérogations mineures, il n'est pas prévu à l'article 145.16 L.A.U. que ce sont les zones «prévues par le règlement de zonage» qui sont visées et que le terme «zone» prévu à cet article se veut donc plus général. À notre avis, cet argument ne peut trouver application compte tenu que le contexte justifie la précision accrue de l'article 145.3 L.A.U. De plus, appliquer un tel raisonnement reviendrait à changer considérablement la portée de l'article 145.9 L.A.U. relatif aux plans d'aménagement d'ensemble qui rattache lui aussi ces plans à l'existence de «zones» et à induire que les zones prévues au plan de zonage ne sont pas nécessairement celles visées par cet article.

Le paragraphe 2 constitue ni plus ni moins le «noyau» de l'article 145.16 L.A.U.

Selon ce paragraphe, le règlement sur les P.I.I.A. devra prévoir les «objectifs applicables» ainsi que les «critères d'évaluation»d'un projet. Alors que l'objectif vise le but recherché par le règlement, le critère doit permettre 
d'évaluer en termes clairs si cet objectif est atteint par le projet proposé ${ }^{144}$. Alors que normalement l'objectif visé est l'intégration d'une construction dans un environnement déjà construit, les critères d'évaluation de cet objectif pourraient contenir des références à la forme et à l'apparence des matériaux utilisés ainsi qu'à la prédominance de certains matériaux dans les constructions environnantes, de manière à limiter, par exemple, l'utilisation de certains matériaux ou d'exiger un emplacement particulier pour les constructions normalement accessoires ou complémentaires à l'usage principal. À cet égard, le paragraphe 3 de cet article vient préciser certains éléments qui pourront être appelés à apparaître dans les plans à être soumis à la municipalité pour évaluation. Il se dégage des paragraphes 2 et 3 que c'est le «caractère» d'un site qui doit être préservé, lequel peut être défini comme étant :

[...] the aggregate of the distinct features that serve to indicate the essential quality or nature of an area. Distinct features include traits, marks, signs, styles and peculiarities with respect to both the physical and social dimensions of an area. Physical character traits refer to such things as streetscape, building types, focal points [such] as a park, etc. Beyond physical traits, however, is a sociocultural identity related to common activities or traditions and lifestyles which define the character of an area ${ }^{145}$.

Pour leur part, les paragraphes 4 et 5 de cet article octroient au conseil municipal le pouvoir d'encadrer la procédure relative à toute demande de permis ou certificat assujettie à la production de plans et des autres documents qu'il exige.

Contrairement au P.A.E., la procédure relative aux P.I.I.A. n'implique aucune modification de la réglementation d'urbanisme. Alors que le P.A.E.

144. Voir 2640-3071 Québec Inc. c. Blainville (Ville de), J.E. 99-834, (C.A.) où la Cour d'appel, citant le juge de première instance, écrivait : «En somme, l'interprétation suggérée par les intimés, n'est pas la seule plausible ou logique, de sorte qu'il subsiste une ambiguïté évidente. Le tribunal est d'avis que cette ambiguïté doit profiter non pas aux intimés mais bien aux requérantes, puisque l'interdiction d'un accès à la propriété des requérantes par le boulevard de la Seigneurie constitue une entrave à leur droit de propriété».

145. Carlton c. City of Toronto Committee of Adjustment, [1990] O.M.B.R. 821; Voir aussi K. Bricker, Planning Issues : The Public Consultation Program, [Toronto] Planning Act Review Committee, [1977], ce rapport ayant trait à la révision du Ontario Planning Act. 
constitue une sorte de règlement d'urbanisme pour une zone donnée, la production d'un P.I.I.A. intervient simplement à l'occasion d'une demande de permis ou certificat. Elle s'ajoute en fait aux exigences déjà prévues aux articles 120 à 122 L.A.U. qui ont trait à l'émission des permis et certificats. À tout événement, si le règlement sur les P.I.I.A. peut être plus sévère que les règlements d'urbanisme, il ne peut cependant l'être moins et risquer de compromettre les objectifs du plan d'urbanisme de la municipalité et, à la limite, ceux du schéma d'aménagement de la M.R.C.

\section{D) L'ÉVALUATION DES PLANS SOUMIS DANS LE CADRE D'UN PLAN D'IMPLANTATION ET D'INTÉGRATION ARCHITECTURALE}

La procédure d'évaluation des plans déposés dans le cadre d'un P.I.I.A. est simple et soumise à très peu de formalisme. Le plus souvent, ce sont les services techniques de la municipalité qui vérifient la demande pour ce qui est des exigences de forme prévues au règlement sur les P.I.I.A. Cette première vérification achevée, c'est le C.C.U., aidé des employés municipaux ou ressources extérieures qui y siègent, qui évaluera la demande quant aux critères de fond. Le C.C.U. présentera ensuite ses recommandations au conseil municipal qui, à cette étape, aura la faculté d'exercer quatre choix, soit :

- $\quad$ approuver la demande avec ou sans modification;

- $\quad$ refuser la demande;

- $\quad$ soumettre les plans produits à une consultation publique suivant les articles 125 à 127 L.A.U. ${ }^{146}$;

- $\quad$ exiger, comme condition d'approbation des plans, que le propriétaire prenne à sa charge le coût de certains éléments des plans, notamment celui des infrastructures ou des équipements, qu'il réalise son projet dans un délai fixé ou qu'il fournisse des garanties financières ${ }^{147}$.

146. L.A.U., supra note 1, art. 145.18 .

147. Ibid., art. 145.20 et L.C.V., supra note 7, art. 470. 
Cette dernière condition ne saurait toutefois être utilisée par un membre du conseil municipal ou du C.C.U. de la municipalité comme prétexte pour extorquer des avantages d'un promoteur désireux de développer un site ${ }^{148}$.

C'est donc par résolution que le conseil municipal approuve ou désapprouve les plans eu égard aux critères prévus au règlement sur les P.I.I.A. Dans le cas où il les désapprouve, sa résolution doit être motivée et faire état des motifs de refus. L'obligation de motiver nous apparaît être une condition essentielle pouvant donner lieu à l'exercice par la Cour supérieure de son pouvoir de surveillance et de contrôle ${ }^{149}$.

Sur présentation de la résolution du conseil municipal et après s'être assuré que le projet est également conforme aux autres dispositions des règlements d'urbanisme, le fonctionnaire désigné devra émettre le permis ou certificat recherché. Une nouvelle demande de P.I.I.A. sera requise si le projet est modifié en cours de réalisation des travaux. Il faut noter que le recours prévu à l'article 227 L.A.U. pourra être mis en oeuvre dans le cas où l'utilisation du sol ou de la construction est incompatible avec un plan approuvé conformément à l'article 145.19 L.A.U. ${ }^{150}$.

148.

149. 150 .
R. c. Bouchard, J.E. 92-451 (C.Q.).

P. Garant, Droit administratif, 4 ., vol.2, Cowansville (Qc), Yvon Blais, 1998, à la p. 308. Voir nos commentaires relativement au contrôle judiciaire des décisions du conseil municipal dans la section relative aux dérogations mineures, section II- F); M.-A. LeChasseur, «L'application du recours selon l'article 227 L.A.U.»(1997), 4 B.D.M. 1-6; M.A. LeChasseur, «La discrétion judiciaire dans l'application de l'article 227 de la Loi sur l'aménagement et l'urbanisme» dans Service de la formation permanente, Barreau du Québec, Développements récents en droit municipal, Cowansville (Qc), Yvon Blais, 1997 aux pp. 1-29; M.-A. LeChasseur, L'injonction déguisée en droit de l'urbanisme - Les ordonnances suivant l'article 227 de la Loi sur l'aménagement et l'urbanisme, Cowansville (Qc), Yvon Blais, 1997; D. Michaud, L'article 227 de la Loi sur l'aménagement et l'urbanisme, thèse de maîtrise en droit, Université Laval, 1997 [non publiée]; D. Michaud, Justice et aménagement du territoire - Le recours prévu à l'article 227 de la Loi sur l'aménagement et l'urbanisme, Sainte-Foy, Union des municipalités régionales de comté et des municipalités locales du Québec Inc., 1997; R.D. Pothier, S. Bilodeau et A. Giguère, Aménagement et urbanisme, Collection Aide Mémoire, Montréal, Wilson \& Lafleur, 1989; G. Rousseau, «La mise en oeuvre du droit de l'aménagement : les recours» dans M. Poirier, dir., Droit québécois de l'aménagement du territoire, Sherbrooke, Éditions R.D.U.S., 1983 aux pp. 366-368; J.-P. St-Amour, «Le dossier d'infraction à un règlement d'urbanisme et la préparation du procès» (1991), 11/2 R.Q.U. 6-8; P. Théroux, «La nature judiciaire des recours en vertu des articles 227 et suivants de la Loi sur l'aménagement et l'urbanisme: 


\section{SECTION V - LES ENTENTES RELATIVES À DES TRAVAUX MUNICIPAUX}

\section{A) ORIGINE}

La réalisation des travaux relatifs aux infrastructures et aux équipements municipaux a longtemps été dévolue aux municipalités. À une certaine époque, il était de coutume de financer à même le prélèvement d'une taxe, une subvention ou par un règlement d'emprunt ce type de travaux. La municipalité concernée veillait elle-même à procéder à l'appel d'offres auprès des entrepreneurs spécialisés et à approprier les deniers nécessaires aux travaux visés.

Peu à peu, s'est développée la pratique opposée qui consistait à requérir du promoteur qu'il finance les «travaux d'infrastructures» et qu'il procède luimême à leur implantation ${ }^{151}$. Toutefois, un tel régime, fruit d'une certaine improvisation, n'était pas sans failles et pouvait entraîner pour la municipalité la prise en charge d'infrastructures parfois de fort mauvaise qualité. De plus, un tel procédé n'était pas sans semer certains doutes, quant à la prise en charge par une municipalité des frais d'aménagement d'un lieu pour le bénéfice d'un tiers entrepreneur en regard des règles strictes prévues à la Loi sur l'interdiction des subventions municipales $^{152}$. De plus, il aurait été illégal et contraire à la Loi sur

Commentaires sur l'arrêt Automobiles Gariépy Inc. c. Village de Saint-Jean-de-Boischatel» dans Service de la formation permanente, Barreau du Québec, Développements récents en droit municipal, Cowansville (Qc), Yvon Blais, 1991 aux pp. 153-163; Ministère des Affaires municipales, Direction générale de l'urbanisme et de l'aménagement du territoire, Recours et sanctions en cas de contravention aux règlements d'urbanisme, 1988, Québec.

151. Constructions Deschênes Ltée c. Coleshill, [1982] C.S. 169; Aménagements Derbec Inc. c. Beloeil (Corporation municipale de la Ville de) (22 janvier 1988), Saint-Hyacinthe 750-05000234-871 (C.S.); Habitat Mon Pays Inc. c. Sainte-Foy (Ville de), J.E. 84-955 (C.S.); Westcliff Investment Ltd. c. Bourdeau, [1984] C.A. 479; Placements Beramla Ltée c. SainteFoy (Ville de), J.E. 93-575 (C.A.); F. Marchand, «Les ententes entre les municipalités et les promoteurs» dans Service de la formation permanente, Barreau du Québec, Développements récents en droit municipal, Cowansville (Qc), Yvon Blais, 1991 aux pp. 81-92.2; S. Sansfaçon, «Ententes relatives aux travaux municipaux - Le point de vue juridique» (1996), 16/2 R.Q.U. 8-14.

152. L.R.Q. c. I-15;Cie Immobilière Viger Ltée c. Lauréat Giguère (1977), 2 R.C.S. 67; Tellier c. Saint-Hyacinthe (Ville de), [1935] R.C.S. 578; Hampstead Land \& Construction Co. Ltd c. Hampstead (Town of) (1928), 44 B.R. 321; Vancouver (City of) c. Registrar of Vancouver 
les travaux municipaux ${ }^{153}$ de permettre au promoteur d'exécuter lui-même les travaux.

C'est dans ce contexte que fut adoptée en 1994 la Loi modifiant la Loi sur l'aménagement et l'urbanisme, laquelle introduisit dans la L.A.U. les articles 145.21 à $145.30^{154}$ inclus dans la section intitulée «Les ententes relatives à des travaux municipaux».

\section{B) LE CHAMP D'APPLICATION}

Suivant l'article 145.21 L.A.U., la municipalité qui désire assujettir la délivrance d'un permis ou d'un certificat à la conclusion préalable d'une entente doit d'abord adopter un règlement à cet effet. Cette entente devra porter «sur la réalisation de travaux relatifs aux infrastructures et aux équipements municipaux et sur la prise en charge ou le partage des coûts relatifs à ces travaux» ${ }^{155}$. Généralement, les travaux municipaux peuvent être désignés comme étant ceux destinés à devenir propriété de la municipalité. C'est elle qui en aura la charge et c'est également à elle que sera dévolu l'entretien des équipements. Vu l'article 116 L.A.U. par lequel la municipalité peut exiger comme condition préalable à la délivrance d'un permis de construction que «le terrain sur lequel doit être érigée la construction projetée [ne] soit adjacent à une rue publique» ${ }^{156}$, il s'avère le plus souvent essentiel pour un promoteur désireux de se conformer à cette condition de conclure d'abord une entente avec la municipalité. La conclusion d'une telle entente ne serait certainement pas nécessaire dans l'éventualité où la réglementation prévoyait que la construction peut être érigée en bordure d'une rue privée ${ }^{157}$. Quant au règlement à proprement parler, puisqu'il s'agit d'un règlement d'urbanisme, il est assujetti à la procédure de consultation publique prévue aux articles 123 et suivants L.A.U.

Land Registration District (1955), 15 W.W.R. 351, 2 D.L.R. 709 (C.A.C.-B.); Herman Brothers Ltd c. Regina (City of), [1976] 6 W.W.R. 85 (Sask. Q.B.).

153. L.R.Q. c. T-14.

154. Loi modifiant la Loi sur l'aménagement et l'urbanisme, L.Q. 1994, c. 32.

155. L.A.U., supra note 1, art. 145.21.

156. Cette exigence fut jugée légale dans l'affaire Westmount (Ville de) c. Weitzman, [1990] R.J.Q. 1762 (C.A.).

157. Gracefield (Corporation du Village de) c. Bériault, [1979] R.P. 279. 


\section{C) LE CONTENU DU RÈGLEMENT}

Selon l'article 145.22 L.A.U., le règlement mentionné à l'article 145.21 doit indiquer :

$1^{\circ}$ toute zone à l'égard de laquelle il s'applique;

$2^{\circ}$ toute catégorie de constructions, de terrains ou de travaux à l'égard de laquelle la délivrance d'un permis de construction ou de lotissement ou d'un certificat d'autorisation ou d'occupation est assujettie à une entente;

$3^{\circ}$ toute catégorie d'infrastructures ou d'équipements visés par l'entente et spécifier, le cas échéant, que l'entente peut porter sur des infrastructures et des équipements, peu importe où ils se trouvent, qui sont destinés à desservir non seulement des immeubles visés par le permis ou le certificat mais également d'autres immeubles sur le territoire de la municipalité;

$4^{\circ}$ les modalités, le cas échéant, suivant lesquelles est établie la part des coûts relatifs aux travaux que le titulaire du permis ou du certificat doit prendre à sa charge selon les catégories de constructions, de terrains, de travaux, d'infrastructures ou d'équipements que le règlement indique.

Le libellé de cet article ne nous apparaît pas autoriser la municipalité à prévoir des règles différentes pour chaque zone visée par le règlement. En effet, le législateur le mentionne expressément lorsqu'il entend permettre que des règles différentes soient adoptées pour chaque zone ${ }^{158}$.

Le règlement devra indiquer quelles sont les catégories de constructions, de terrains ou de travaux assujettis à une entente. Il pourra s'agir, par exemple, du cas des nouvelles subdivisions ou d'un développement résidentiel.

Il devra également prévoir les catégories d'infrastructures ou d'équipements visés par l'entente, que l'on pense seulement aux aqueducs, égouts, bordures, trottoirs ou aux fondations des rues.

158. Voir par exemple, L.A.U., ibid., art. 145.17. Voir également A. Langlois, «Les ententes avec les promoteurs concernant des travaux municipaux», Municipalité, juin-juillet 1995 aux pp. 18-21. 
La seconde partie du paragraphe 3 de l'article 145.22 L.A.U. vise le cas où des équipements supplémentaires ou «surdimensionnés» sont implantés pour desservir le site qui fait l'objet de travaux municipaux et qui, incidemment, desservent d'autres immeubles situés sur le territoire de la municipalité ou qui seront éventuellement desservis par les infrastructures «surdimensionnées». Cette disposition visait plus particulièrement à permettre à la municipalité d'impliquer les free riders (tiers bénéficiaires) dans le processus d'exécution et de paiement des travaux.

Quant aux paragraphes 4 et 5 de cet article, ils permettent à la municipalité de prévoir la répartition des coûts relatifs aux travaux que tout bénéficiaire doit prendre à sa charge ainsi que les modalités suivant lesquelles est établie et perçue cette quote part et de fixer le taux d'intérêt payable sur un versement devenu exigible. Encore une fois, les free riders sont visés par la grande latitude octroyée à la municipalité dans la détermination de la part des coûts attribuable à ces bénéficiaires. Il faut noter que ces bénéficiaires demeurent assujettis à l'obligation de payer leur quote-part en vertu du règlement, même après le passage de la date limite où la municipalité doit rembourser au promoteur les quotes-parts impayées selon l'article 145.23 (7) L.A.U.

L'entente qui prévoit le paiement d'une quote-part par les bénéficiaires des travaux visés au paragraphe $5^{\circ}$ du premier alinéa de l'article 145.22 doit identifier dans une annexe à cette entente, les immeubles qui assujettissent les bénéficiaires des travaux à cette quote-part ou doit mentionner tout critère permettant de les identifier. La municipalité peut modifier par résolution cette annexe pour la tenir à jour ou y ajouter tout immeuble qui assujetti un bénéficiaire des travaux à la quote-part ${ }^{159}$.

Enfin, le paragraphe 6 autorise le conseil municipal à assujettir la délivrance d'un permis de construction ou de lotissement ou d'un certificat d'autorisation ou de construction demandé par un bénéficiaire de travaux visés au paiement préalable de toute partie de sa quote-part ou à la production de toute garantie que le règlement détermine. 
La municipalité, bien qu'elle puisse toujours se prévaloir du régime antérieur par lequel elle pouvait exiger des garanties de toute personne requérant d'elle qu'elle effectue des travaux ${ }^{160}$, aura l'obligation d'adopter le règlement prévu à l'article 145.22 L.A.U. dans trois cas particuliers. En premier lieu, le fait de confier au promoteur la réalisation de certains travaux nécessite l'adoption d'un tel règlement. Il s'agit en fait de l'innovation apportée par ce nouveau processus qui «légalise» une telle pratique. En second lieu, le règlement s'avère nécessaire dans l'optique où une quote-part des travaux est payable par des tiers bénéficiaires des travaux (autre que le promoteur) et qu'un tel paiement se fait à l'extérieur du cadre d'un règlement d'emprunt. En dernier lieu, le règlement sera également nécessaire pour la conclusion de toute entente en vertu de l'article 145.23 L.A.U. Il faut noter que conformément à l'article 145.30 L.A.U., le dépôt d'un avis de motion en vue d'adopter un tel règlement entraîne un «effet de gel» sur la délivrance des permis de construction ou de lotissement ou des certificats d'autorisation ou d'occupation qui seront assujettis, suivant l'entrée en vigueur du règlement, à la conclusion d'une entente.

\section{D) LE CONTENU DE L'ENTENTE}

Selon l'article 145.23 L.A.U., l'entente qui doit être conclue pour les catégories de constructions, de terrains ou de travaux visés par le règlement doit, notamment, prévoir les éléments suivants :

$1^{\mathrm{o}}$ la désignation des parties;

$2^{\circ}$ la description des travaux et la désignation de la partie responsable de tout ou partie de leur réalisation;

$3^{\circ}$ la date à laquelle les travaux doivent être complétés, le cas échéant, par le titulaire du permis ou du certificat;

$4^{\circ}$ la détermination des coûts relatifs aux travaux à la charge du titulaire du permis ou du certificat;

$5^{\circ}$ la pénalité recouvrable du titulaire du permis ou du titulaire du certificat en cas de retard à exécuter les travaux qui lui incombent;

$6^{\circ}$ les modalités de paiement, le cas échéant, par le titulaire du permis ou du certificat des coûts relatifs aux travaux et l'intérêt payable sur un versement exigible; 
$7^{\circ}$ les modalités de remise, le cas échéant, par la municipalité au titulaire du permis ou du certificat de la quote-part des coûts relatifs aux travaux payable par un bénéficiaire des travaux; les modalités de remise de la quote-part doivent prévoir une date limite à laquelle la municipalité doit rembourser, le cas échéant, au titulaire du permis ou du certificat une quote-part non payée;

$8^{\circ}$ les garanties financières exigées du titulaire du permis ou du certificat.

L'entente devra donc prévoir que les travaux sont en partie ou en entier réalisés soit par la municipalité, soit par le promoteur et déterminer un échéancier pour leur réalisation. De plus, l'entente devra déterminer la part des coûts $(50 \%, 75 \%, 100 \%, \ldots)$ reliés au projet qui sont à la charge du promoteur. Dans l'éventualité où un surdimensionnement est requis pour desservir des immeubles visés ou non spécifiquement visés par le permis ou certificat, l'entente pourra prendre en considération ces coûts supplémentaires et prévoir un mécanisme de répartition entre la municipalité et le promoteur.

Bien que la municipalité n'ait pas l'obligation d'assumer une quote-part des travaux, si elle décide de le faire, elle devra nécessairement prévoir un mode de financement desdits travaux, qu'il s'agisse des travaux du projet ou des travaux de surdimensionnement. Cette obligation découle de l'article 145.26 L.A.U. qui se lit comme suit :

145.26. Les articles 1 à 3 de la Loi sur les travaux municipaux (chapitre t-14) ne s'appliquent pas aux travaux exécutés conformément à une entente. Toutefois, les règles prévues par cette loi relativement au mode de financement de ces travaux par la municipalité s'y appliquent.

La municipalité dispose donc, pour ce faire, de quatre modes de financement :

une subvention pour le type de travaux concernés;

le fonds général de la municipalité;

- $\quad$ le décret d'une taxe spéciale; ou

- l'adoption d'un règlement d'emprunt (celui-ci prévoira nécessairement une taxe spéciale, une tarification, une 
participation du fonds général ou l'appropriation du montant d'une subvention);

Outre la participation financière de la municipalité et du promoteur, tout bénéficiaire des travaux pourra être tenu de prendre à sa charge une part des coûts de ces derniers. Il faudra donc déterminer, dans l'entente, les modalités de paiement et de perception de la quote-part payable par le bénéficiaire et fixer le taux de l'intérêt payable sur le montant exigible ${ }^{161}$.

Le paiement d'une quote-part des coûts relatifs aux travaux payable par un bénéficiaire devra servir aux fins déterminées dans l'entente. Celle-ci devra prévoir les modalités de remise au promoteur qui a pris à sa charge une partie des coûts reliés au projet ou au surdimensionnement ${ }^{162}$. Cependant, ces modalités de remise sont assujetties à la fixation d'une date limite à laquelle la municipalité doit rembourser au promoteur les quotes-parts non payées. Cette disposition vise évidemment à éviter que les argents soient escomptés dans un temps indéfini, au détriment du promoteur.

Il s'agit là d'une importante prise en charge par la municipalité de coûts qu'elle n'avait pas nécessairement prévu devoir payer. C'est pourquoi il est impératif que la date limite soit déterminée à même un échéancier de développement réaliste qui assurera à la municipalité une perception régulière desdites quotes-parts et lui évitera la surprise de devoir financer des infrastructures desservant un projet de développement demeuré à l'état d'embryon. À tout événement, cette date limite n'a cours qu'entre le promoteur et la municipalité et n'influence aucunement le paiement de la quote-part par les tiers bénéficiaires identifiés à l'annexe à l'entente qui demeure toujours due ${ }^{163}$.

\section{CONCLUSION}

L'intégration ponctuelle à la L.A.U. de mécanismes de contrôle discrétionnaires a considérablement transformé le droit de l'urbanisme

161. L.A.U., supra note 1, art. 145.22 (5).

162. Ibid., art. 145.24 (7) et 145.25.

163. Ibid., art. 145.24. 
québécois. Bien que la philosophie sous-jacente à la technique de zonage retenue en droit québécois soit rigide et peu conviviale, le rapprochement des dernières années avec la tendance anglo-saxonne l'a grandement actualisée.

Le législateur, plutôt que d'adapter la procédure de modification de la réglementation d'urbanisme qui, de toute évidence, n'est pas un modèle de simplicité, a plutôt opté pour l'adoption de mécanismes complémentaires dont l'effet indirect est de contourner «l'opacité de notre régime de zonage».

Les règles relatives aux dérogations mineures sont un exemple évident de cet effet. Bien que l'on ne peut que se réjouir de l'utilisation d'un vocabulaire très libéral dans la détermination de ces règles, il n'en demeure pas moins qu'une certaine confusion demeure quant à leur application vu l'ambiguïté de la terminologie utilisée. À cet effet, nous soulignons la difficulté qui demeure quant à l'interprétation à donner à l'expression «densité d'occupation du sol».

Quant à la technique des P.A.E., bien qu'elle n'ait été intégrée à la L.A.U. que tardivement, elle constitue sans contredit une des démarches les plus louables du législateur. Bien que comme la technique des P.I.I.A. elle expose le citoyen à un risque plus élevé d'abus ou d'arbitraire, elle s'applique toutefois difficilement aux zones où il y a morcellement des propriétés.

Quant aux ententes avec les promoteurs, la loi contient une énumération assez précise des matières pouvant faire l'objet de conditions et d'ententes. Après lecture de la jurisprudence anglaise en cette matière, il appert que le législateur québécois a retenu des principes valables bien qu'encore une fois il ait, à notre avis, inutilement tardé à porter à maturité un tel système qui s'imposait depuis longtemps.

Il sera intéressant de suivre les prochains développements législatifs en matière de réglementation discrétionnaire. La tendance est indubitablement au dynamisme de la réglementation d'urbanisme. Peu à peu, nous nous rapprochons du Development Control du droit anglais qui constitue une alternative intéressante au zonage tel que nous le connaissons. 
Une réforme menée en ce sens ne pourrait toutefois se faire sans une révision complète des modes de consultation et de participation des citoyens à l'adoption et à la modification des règlements. En cette aire de réforme, il y a tout lieu de souhaiter que de tels changements soient plus près de nous que le contraire... 